A mesoscopic model of nucleation and Ostwald ripening/stepping: Application to the silica polymorph system

G. Ozkan, and P. Ortoleva

Citation: The Journal of Chemical Physics 112, 10510 (2000);

View online: https://doi.org/10.1063/1.481685

View Table of Contents: http://aip.scitation.org/toc/jcp/112/23

Published by the American Institute of Physics

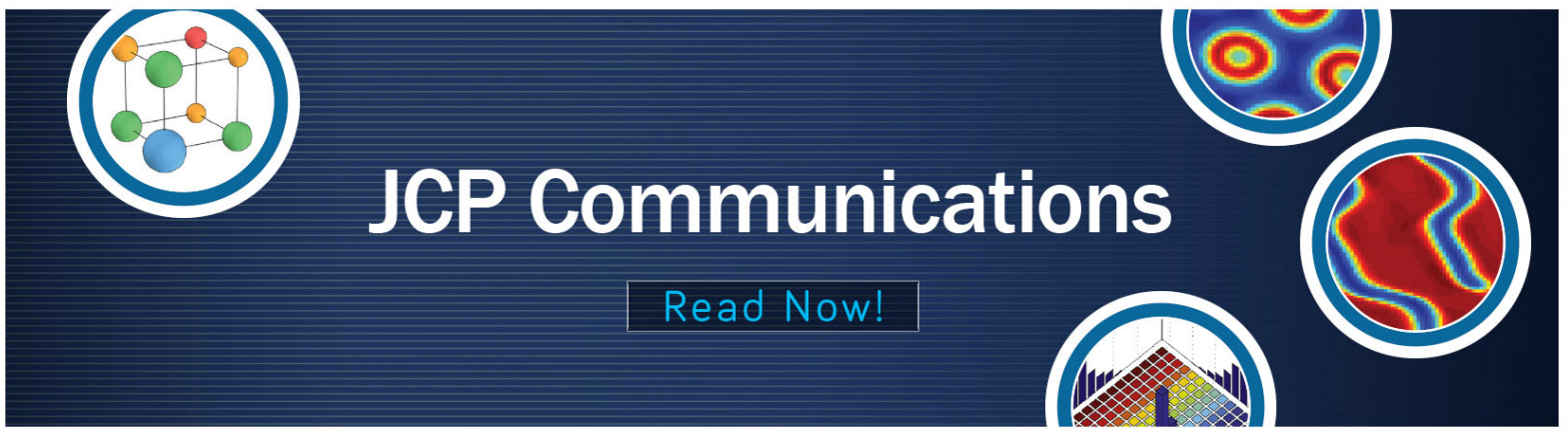




\title{
A mesoscopic model of nucleation and Ostwald ripening/stepping: Application to the silica polymorph system
}

\author{
G. Ozkan ${ }^{a)}$ and P. Ortoleva \\ Laboratory for Computational Geodynamics, Department of Chemistry, Indiana University, \\ Bloomington, Indiana 47405
}

(Received 10 September 1999; accepted 20 March 2000)

\begin{abstract}
Precipitation is modeled using a particle size distribution (PSD) approach for the single or multiple polymorph system. A chemical kinetic-type model for the construction of the molecular clusters of each polymorph is formulated that accounts for adsorption at a heterogeneous site, nucleation, growth, and Ostwald ripening. When multiple polymorphs are accounted for, Ostwald stepping is also predicted. The challenge of simulating the 23 order of magnitude in cluster size (monomer, dimer, $\ldots, 10^{23}$-mer) is met by a new formalism that accounts for the macroscopic behavior of large clusters as well as the structure of small ones. The theory is set forth for the surface kinetic controlled growth systems and it involves corrections to the Lifshitz-Slyozov, Wagner (LSW) equation and preserves the monomer addition kinetics for small clusters. A time independent, scaled PSD behavior is achieved both analytically and numerically, and the average radius grows with $R_{\text {ave }} \propto t^{1 / 2}$ law for smooth particles. Applications are presented for the silica system that involves five polymorphs. Effects of the adsorption energetics and the smooth or fractal nature of clusters on the nucleation, ripening, and stepping behavior are analyzed. The Ostwald stepping scenario is found to be highly sensitive to adsorption energetics. Long time scaling behavior of the PSD reveals time exponents greater than those for the classical theory when particles are fractal. Exact scaling solutions for the PSD are compared with numerical results to assess the accuracy and convergence of our numerical technique. (C) 2000 American Institute of Physics. [S0021-9606(00)70123-1]
\end{abstract}

\section{INTRODUCTION}

Precipitation typically involves four distinct processesadsorption at a heterogeneous site, nucleation, Ostwald ripening, and Ostwald stepping. These processes underlie many key phenomena associated with chemical processing, as well as, in nature (weathering, sedimentary, metamorphic, igneous, and other environments). Precipitation phenomena in nature include the nucleation and ripening of new solid phases, selected precipitation of preferred polymorphs, Lisegang banding, agates, and igneous orbicules. ${ }^{1-5}$ While the importance of these phenomena has long been recognized, their simulation has been limited due to numerical difficulties involved in describing the growth of particles over 23 orders of magnitude in size (i.e., from monomer to $\mathrm{cm}$-scale crystals). A complete model of precipitation must therefore integrate the microscopic, mesoscopic, and macroscopic levels of description.

Several approaches have been used to model precipitation kinetics. ${ }^{6-9}$ In nucleation-growth studies, a critical nucleus size is defined such that further growth is a free energy reducing process and thus a supracritical cluster may grow to macroscopic dimensions. Studies on the nucleation stage focus on the behavior of clusters smaller than this critical size and on calculating the total number of supercritical clusters but not on the transition from the nucleation stage to that of macroscopic particles.

Studies of the coarsening of precipitates after the nucle-

\footnotetext{
${ }^{a}$ Current address: Department of Mathematics, Bilkent University, Bilkent, Ankara 06533, Turkey.
}

ation stage are usually based on the theory of Lifshitz and Slyozov $^{10}$ and Wagner, ${ }^{11}$ and its modifications. ${ }^{12-15}$ The classical and modified versions of the LSW theory yield a power law relation between the mean particle size and time at long times and a scaling form for the particle size distribution (PSD). The low volume fraction theories of Marquesse and Ross ${ }^{14}$ and Tokuyama and Kawasaki,${ }^{16}$ although each employed a different statistical averaging procedure, all predict a scaled particle size distribution which is broader than the LSW form.

In this study, we developed a model of precipitation phenomena which uses the same chemical rate formulation for both the early nucleation stage (the kinetics of monomer adsorption, dimer creation, growth of small surface-attached clusters) and the growth and ripening stage to create macroscopic crystals. In the model, nucleation is initiated as an adsorption event. Further monomer addition results in the formation of small clusters whose dynamics becomes increasingly independent of the adsorption site properties as they grow. However, we do not separately treat the sub- and supracritical clusters. Rather, we apply the same chemical rate equation over the whole size range by using a sizedependent rate coefficient and free energy, giving a unity and continuity to the theory.

Our formulation adds a higher order derivative term to the LSW theory that introduces a diffusive-type behavior with respect to the particle size axis; this tends to broaden the PSD. For simplicity here, we refer to all PSD evolution theories in the macroscopic particle limit to the LSW theories. In the present work, however, we focused on the reaction- 
limited case, and not the diffusion-limited case of the classic LSW theory. A second contribution is an efficient numerical simulation technique that allows for the solving of these equations for 23 orders of magnitude in cluster size. Finally, we simulate PSD dynamics in multiple polymorph systems to investigate Ostwald stepping and the commonly observed selected precipitation of higher free energy solids or the skipping of intermediate free energy ones. We illustrate the relation of nucleation site energetics to the latter.

The present study focused on the $\mathrm{SiO}_{2}$ system due to its importance in many geologic and engineering processes and the availability of ample rate and thermodynamic data. ${ }^{17-19}$ A model is set forth of the precipitation of $\mathrm{SiO}_{2}$ into a set of polymorphs which compete for the shared pool of $\mathrm{SiO}_{2}$. Particles of the same polymorph but of different size also compete to yield ripening of the PSD of each polymorph. The resulting model thus incorporates Ostwald ripening and stepping. The approach is easily generalized to other mineralization (carbonates, feldspars, etc.).

The formulation of our model is given in the next three sections. Numerical simulations based on our model for the $\mathrm{SiO}_{2}$ system of initially amorphous silica are discussed in Sec. V. These simulations show the selective nucleation of some polymorphs and the competitive evolution of polymorphs as $\mathrm{SiO}_{2}$ cascades from the amorphous state initially in the system, through several polymorphs, and finally to the long time dominance by the lowest free energy phase, quartz (e.g., Ostwald stepping). After the stepping phase is completed, larger quartz crystals grow at the expense of the smaller ones. A power law for the mean diameter is demonstrated. The PSD attains a LSW limiting scaled form in the ripening phase. The simulated and exact scaled PSD are compared to evaluate the accuracy of the numerical methods. Conclusions are drawn in Sec. VI. The detailed formulations for the model and the $\mathrm{SiO}_{2}$ data used in the numerical simulations are discussed in the Appendices.

\section{FORMULATION}

The model developed here is based on the classic chemical kinetic theory of nucleation through monomer addition modified for heterogeneous systems and which integrates the chemical kinetic and continuous (macroscopic) particle growth as suggested in Ref. 1. Modifications of the free energy are introduced to account for the heterogeneous nature of initiation and early small cluster growth as well as surface energy for larger clusters.

The assumed kinetics of heterogeneous nucleation and growth is based on monomer addition discussed here in terms of the $\mathrm{SiO}_{2}$ system due to its importance in many geologic and engineering processes and the availability of ample rate and thermodynamic data, ${ }^{17-19}$ but the approach is easily generalized to other mineralization. Let $S_{n}^{m}$ be a cluster of polymorph $m$ with $n \mathrm{SiO}_{2}$ units on a heterogeneous site. The monomer addition reaction is taken to be

$$
S_{n}^{m}+\mathrm{SiO}_{2}(\mathrm{aq}) \Leftrightarrow S_{n+1}^{m} .
$$

Let $\xi_{n}^{m}$ be the fraction of sites occupied by $m, n$-cluster in a macrovolume element having $\eta_{T}$ identical sites per unit vol- ume. The total number of sites in the macrovolume element is assumed to be constant during the evolution; it is the sum of the numbers of clusters for every polymorph cluster and the number of unoccupied surface sites available for nucleation. During evolution the number of sites occupied by various $m, n$-clusters and the shared pool of $\mathrm{SiO}_{2}(\mathrm{aq})$ are constantly shifting due to competition among polymorphs and among different size clusters of a given polymorph. The fraction of empty sites, $\xi_{0}$, is given in terms of the fractions of $m, n$-cluster-occupied sites via

$$
\xi_{0}=1-\sum_{m=1}^{M} \sum_{n=1}^{\infty} \xi_{n}^{m}
$$

where $M$ is the number of polymorphs.

The dynamics of the site occupation fractions is assumed to take the chemical kinetic form

$$
\begin{aligned}
& \frac{d \xi_{0}}{d t}=-\sum_{m=1}^{M} W_{0}^{m}, \\
& \frac{d \xi_{n}^{m}}{d t}=W_{n-1}^{m}-W_{n}^{m} \quad n=1,2, \ldots, \infty \quad m=1,2, \ldots, M .
\end{aligned}
$$

The rates $W_{n}^{m}$ are assumed to take the mass action form

$W_{0}^{m}=k_{0}^{m}\left(K_{0}^{m} c \xi_{0}-\xi_{1}^{m}\right), \quad W_{n}^{m}=k_{n}^{m}\left(K_{n}^{m} c \xi_{n}^{m}-\xi_{n+1}^{m}\right)$,

for size-dependent rate coefficient $k_{n}^{m}$, equilibrium constant $K_{n}^{m}$, and $\mathrm{SiO}_{2}$ (aq) molar concentration $c$. The data for silica polymorphs are described in the next section.

These equations describe the dynamics of the site occupancy probabilities (fractions). The $\xi_{n}^{m}$ are the PSD's of the polymorphs in the present formulation. The kinetics depends on the $\mathrm{SiO}_{2}(\mathrm{aq})$ concentration variation with time in the macrovolume element. This variation is obtained by equating the rate of change of total $\mathrm{SiO}_{2}$ units in the macrovolume (both in aqueous phase or in cluster form) to the total rate of flux into the volume. The $\mathrm{SiO}_{2}(\mathrm{aq})$ concentration thus evolves via

$\frac{\partial \phi c}{\partial t}+\frac{\partial}{\partial t}\left(\frac{\eta_{T}}{N^{0}} \sum_{m=1}^{M} \sum_{n=1}^{\infty} n \xi_{n}^{m}\right)=\left(\begin{array}{c}\text { Total } \mathrm{SiO}_{2} \text { flux into } \\ \text { the volume element }\end{array}\right)$

where $\phi$ is the porosity and $N^{0}$ is Avogadro's number. If $V_{\text {in }}$ is the volume of inert solid in the medium, the porosity is calculated by subtracting the volume fraction of all polymorphs and inert solid from unity,

$$
\phi=1-\frac{V_{\text {in }}}{V_{T}}-\eta_{T} \sum_{m=1}^{M} \frac{1}{N^{0}} \sum_{n=1}^{\infty} \bar{V}_{n}^{m} \xi_{n}^{m},
$$

where $\bar{V}_{n}^{m}$ is the molar volume of a polymorph $m$ cluster of $n$ units and $V_{T}$ is the volume of the macrovolume element.

Equations (2.3) - (2.6) conclude the chemical kinetic formulation of the PSD dynamics of polymorph growth via monomer addition. These equations constitute a set of nonlinear differential equations for $c$, the PSDs of all polymorphs, the fraction of empty sites and the porosity. These equations are solved by Newton-Raphson algorithm with second order time approximation. 
To complete the formulation, we must specify the $n$-dependence of $\bar{V}_{n}^{m}, k_{n}^{m}$, and $K_{n}^{m}$. We assumed that the volume of clusters is proportional to the cluster number. The phenomenology of $k_{n}^{m}$ and $K_{n}^{m}$ are developed further in Sec. IV.

\section{THE MESOSCOPIC TRANSITION FROM NUCLEUS TO MACROPARTICLE}

The chemical kinetic formulation bridges the transition from monomer and dimer to macroparticle $\left(10^{23}\right.$-mer). However, there are several challenges presented by this formulation. First, one must solve on the order of $10^{23}$ equations because the cluster size ranges over this scale. Second, the $k_{n}^{m}$ increase as surface area (as $n^{2 / 3}$ for small particles) and hence become extremely large as $n \rightarrow 10^{23}$. The latter situation presents us with a problem in stiff differential equations, i.e., equations with coefficients that differ by many orders of magnitude. The result is that to maintain accuracy, one must know the factors $K_{n}^{m} c \xi_{n}^{m}-\xi_{n+1}^{m}$ to an accuracy of order $1 / k_{n}^{m}$. Thus even if one could solve $10^{23}$ equations numerically, the accuracy required would make the calculation unfeasible.

The resolution of these difficulties resides in the realization that very large clusters act like macroparticles and thus obey macroscopic-type growth equation. A quartz crystal of $0.1 \mu \mathrm{m}$ diameter is modeled as $1.4 \times 10^{7}$-mer cluster. For cm-scale crystals, the number $n$ of $\mathrm{SiO}_{2}$ units goes up to $10^{23}$. Also, ripening makes PSD dynamics a type of moving boundary problem, i.e., the range of cluster sizes grows with time. This makes the computational range infinitely large, with infinite number of equations. These difficulties are overcome by dividing the cluster size domain into two parts at a cluster number $N$ (say, $N=100$ ). For $n<N$, the discrete chemical kinetic formulation given in Eqs. (2.3) and (2.4) is applied. But for larger clusters, we transform the discrete kinetic equations into a continuous form by introducing the continuous site fraction, $\widetilde{\xi}$, and continuous cluster volume fraction, $y$, via

$$
\begin{aligned}
& \xi_{n}^{m}(t)=\int_{n-1 / 2}^{n+1 / 2} \widetilde{\xi}^{m}(n, t) d n, \\
& y^{m}(n, t)=n \widetilde{\xi}^{m}(n, t) .
\end{aligned}
$$

The quantities $\tilde{\xi}$ and $y$ are assumed to be smooth functions of $n$ so that they and the first two derivatives with respect to $n$ are well defined for all $n>N$.

When $n>N$, we assume that for $|\delta| \leqslant 1$, we may write

$$
y^{m}(n+\delta, t)=y^{m}(n, t)+\frac{\partial y^{m}}{\partial n}(n, t) \delta+\frac{1}{2} \frac{\partial^{2} y^{m}}{\partial n^{2}}(n, t) \delta^{2} .
$$

With this specific meaning of smoothness of $y^{m}$ with respect to $n$, insertion of these definitions and Eq. (3.3) into (2.3) yields the continuous equation for the PSD,

$$
\frac{\partial y^{m}}{\partial t}+\frac{\partial}{\partial x}\left[v^{m} y^{m}-D^{m} \frac{\partial y^{m}}{\partial x}\right]=0, \quad n \geqslant N,
$$

where $x=\ln (n)$, and $v$ and $D$ are related to the chemical parameters via

$$
\begin{aligned}
& v^{m}(n, c)=\frac{k_{n-1 / 2}^{m}}{n}\left[\left(1+\frac{1}{2 n}\right) K_{n-1 / 2}^{m} c-\left(1-\frac{1}{2 n}\right)\right], \\
& D^{m}(n, c)=\frac{k_{n-1 / 2}^{m}}{2 n^{2}}\left(K_{n-1 / 2}^{m} c+1\right) .
\end{aligned}
$$

The derivation of these results is given in Appendix A. Note that Eq. (3.4) yields the classical LSW equation when the $1 / n^{2}$ term in $v$ is ignored and the diffusionlike $D$ term is neglected. Thus Eq. (3.4) $\rightarrow \mathrm{LSW}$ as $n \rightarrow \infty$. However, for finite $n$ the PSD is broader than that of the LSW due to diffusive spreading from $D \neq 0$.

The boundary conditions between the discrete and continuous formulations are obtained from the continuity of the $\tilde{\xi}$ or $y$ and from Eq. (2.2). At the interface, the boundary condition to be imposed on Eq. (3.4) is

$$
\left[v^{m} y^{m}-D^{m} \frac{\partial y^{m}}{\partial x}\right]_{x=x_{0}=\ln \{N-1 / 2\}}=W_{N-1}^{m},
$$

and for Eq. (2.3) the boundary condition is

$$
\xi_{N}^{m}(t)=\xi_{N-1}^{m}(t)+2 \frac{y^{m}(N-1 / 2, t)}{N-1 / 2} .
$$

With this discrete and continuous formulation, numerical simulation of the mesoscopic transition becomes feasible as the solution of the following integrated problem:

$$
\begin{aligned}
& \frac{d \xi_{0}}{d t}=-\sum_{m=1}^{M} W_{0}^{m}, \\
& \frac{d \xi_{n}^{m}}{d t}=W_{n-1}^{m}-W_{n}^{m} \quad n=1,2, \ldots, N-1, \\
& \frac{\partial y^{m}}{\partial t}+\frac{\partial}{\partial x}\left[v^{m} y^{m}-D^{m} \frac{\partial y^{m}}{\partial x}\right]=0, \quad x \geqslant \ln (N-1 / 2)
\end{aligned}
$$

with

$W_{0}^{m}=k_{0}^{m}\left(K_{0}^{m} c \xi_{0}-\xi_{1}^{m}\right)$,

$W_{n}^{m}=k_{n}^{m}\left(K_{n}^{m} c \xi_{n}^{m}-\xi_{n+1}^{m}\right)$,

$$
\begin{aligned}
\frac{\partial \phi c}{\partial t} & +\frac{\partial}{\partial t}\left(\frac{\eta_{T}}{N^{0}} \sum_{m=1}^{M} \sum_{n=1}^{N-1} n \xi_{n}^{m}+\frac{\eta_{T}}{N^{0}} \sum_{m=1}^{M} \int_{x_{0}}^{\infty} e^{x} y^{m}(x, t) d x\right) \\
& =0
\end{aligned}
$$

where

$$
\begin{aligned}
\phi= & 1-\frac{V_{\mathrm{in}}}{V_{T}}-\eta_{T} \sum_{m=1}^{M} \frac{1}{N^{0}} \sum_{n=1}^{N-1} \bar{V}^{m} n \xi_{n}^{m} \\
& -\eta_{T} \sum_{m=1}^{M} \frac{1}{N^{0}} \int_{x_{0}}^{\infty} \bar{V}^{m} e^{x} y^{m}(x, t) d x .
\end{aligned}
$$


TABLE I. Silica polymorph data.

\begin{tabular}{clccl}
\hline \hline $\begin{array}{c}\text { Mineral } \\
\text { number }\end{array}$ & Mineral species & $\begin{array}{c}V_{m}{ }^{\mathrm{a}} \\
\left(\mathrm{cm}^{3} \mathrm{~mol}^{-1}\right)\end{array}$ & $\begin{array}{c}\text { Surface free } \\
\text { energy } \\
\sigma_{i}\left(\mu \mathrm{J} \mathrm{cm}^{-2}\right)\end{array}$ & $\begin{array}{c}\text { Log dissolution rate } \\
q_{i}\left(\mathrm{~mol} \mathrm{~m}^{-2} \mathrm{~s}^{-1}\right)\end{array}$ \\
\hline 1 & Amorphous silica & 29.0 & $4.5^{\mathrm{b}}$ & $-0.369-7.890 \times 10^{-4} T-3438 / T$ \\
2 & $\beta$-cristobalite & 27.38 & $6.01^{\mathrm{c}}$ & $-0.936-3392 / T$ \\
3 & $\alpha$-cristobalite & 25.74 & $8.43^{\mathrm{c}}$ & $-0.739-3586 / T$ \\
4 & Chalcedony & 22.688 & $10.65^{\mathrm{c}}$ & $1.744-2.847 \times 10^{-3} T-4189 / T$ \\
5 & $\alpha$-quartz & 22.688 & $12^{\mathrm{b}}$ & $1.174-2.028 \times 10^{-3} T-4158 / T$ \\
\hline \hline
\end{tabular}

${ }^{a}$ Thermodynamic data from Ref. 18.

${ }^{\mathrm{b}}$ Surface free energies taken from Ref. 17.

${ }^{\mathrm{c}}$ Surface free energies estimated by Eq. (B4).

${ }^{\mathrm{d}}$ Experimental dissolution rates taken from Ref. 19. $T$ is in K.

In the numerical analysis of this system, the continuous range is truncated at a sufficiently large value of $x$ such that $y$ is negligible beyond this value. The continuous problem is solved by finite element discretization, i.e., the $x$ axis between $\ln N$ and the cutoff value is divided into $N_{F}$ equal segments. With this, the system size is reduced to the number of finite elements, $N_{F}$, plus $N$, with $N+N_{F} \ll 10^{23}$ (typically $N+N_{F}$ is taken to be between 300 and 4000 in the results presented here, yielding an improvement of $10^{20}$ in the size of the problem order that stated in Sec. II). Finally, as $k_{n}^{m}$ is always divided by at least one factor of $n$ in the equation of motion for $y$, the stiffness of the problem has also been removed. The system is solved by the NewtonRaphson algorithm with an upwinding scheme for the continuous part.

\section{EQUILIBRIUM AND RATE PHENOMENOLOGIES}

The size dependent equilibrium constant is obtained from dilute solution equilibrium equation. At equilibrium, assuming a dilute aqueous $\mathrm{SiO}_{2}$ solution,

$$
g_{n}^{m}+\mu_{\mathrm{SiO}_{2}(\mathrm{aq})}^{* *}+R T \ln \left(\frac{1}{K_{n}^{m}}\right)=g_{n+1}^{m},
$$

where $\mu_{\mathrm{SiO}_{2}}^{* *}{ }_{(\mathrm{aq})}$ is the reference potential of $\mathrm{SiO}_{2}(\mathrm{aq}), R$ is the gas constant, $T$ is absolute temperature, and $g_{n}^{m}$ is the free energy of an $n$-mer of polymorph $m$ (referred as $m, n$ cluster in this document). The $\left(K_{n}^{m}\right)^{-1}$ is the saturation $\mathrm{SiO}_{2}(\mathrm{aq})$ concentration of the $m, n$ cluster. The free energy of an $n$-mer is classically related to the surface free energy and bulk free energy via

$$
\left(g_{n}^{m}\right)^{\mathrm{cl}}=a_{m} n+b_{m} n^{2 / 3},
$$

where $a_{m}$ is the Gibbs free energy of bulk $m$ per $\mathrm{SiO}_{2}$ unit (i.e., the molar free energy divided by Avogadro's number). The $b_{m}$ factor is related to the surface free energy (free energy per area) $\sigma_{m}$ via

$$
b_{m}=\beta \sigma_{m}\left(\frac{\bar{V}_{m}}{N^{0}}\right)^{2 / 3},
$$

where $N^{0}$ is Avogadro's number and $\beta$ is a geometry factor relating molar volume $\bar{V}_{m}$ to grain surface [for spherical grains $\beta=(36 \pi)^{1 / 3}$.
The above formulation must be supplemented with a term that reflects the adsorption of $\mathrm{SiO}_{2}$ units onto heterogeneous nucleation sites in the sediment. One might expect this to be proportional to the number of adsorbed $\mathrm{SiO}_{2}$ units. Hence, for small $n$ we assume the independent adsorption energy behavior $g_{n}^{m} \sim d_{m} n$ for a coefficient $d_{m}$ related to the binding free energy of a $\mathrm{SiO}_{2}$ unit to the site surface. To create an interpolation between the small and larger $n$ formulas, consider the ansatz

$$
g_{n}^{m}=\psi(n) d_{m} n+(1-\psi(n))\left(g_{n}^{m}\right)^{\mathrm{cl}},
$$

for weighting factor $\psi(n)$ that varies between 1 and 0 as $n$ varies between 1 and infinity. For concreteness, in this study we assume

$$
\psi(n)=\frac{1}{1+(n-1) / n_{0}}
$$

for parameter $n_{0}$ that fixes the transition number of $\mathrm{SiO}_{2}$ units beyond which the $\left(g_{n}^{m}\right)^{\mathrm{cl}}$ term should dominate. These formulas determine the $n$-dependence of the saturation $\mathrm{SiO}_{2}$ (aq) concentration for $m, n$-mer.

The adsorption effect also included in the rate coefficient in a similar way. For small clusters the rate coefficient is proportional to the area of the nucleation sites and as the cluster grows, the crystal surface area becomes dominant for the kinetics rate. In our model, we used the same weighting factor $\psi(n)$ for interpolation of rate coefficients as

$$
k_{n}^{m}=\bar{k}_{m} \phi\left[\psi(n) A_{0}+(1-\psi(n)) \beta\left(\frac{\bar{V}_{m}}{N^{0}}\right)^{2 / 3} n^{2 / 3}\right],
$$

where $\bar{k}_{m}$ is the rate coefficient per surface area for polymorph $m$ and $A_{0}$ is the average area of the nucleation sites. The porosity, $\phi$ (i.e., the volume fraction of fluid with aqueous species) is included in the rate coefficient expression to model the effect of volume fractions of crystals in chemical rate. As the volume fraction of solids increases the kinetic rate decreases since the contact area of solids with liquids decreases.

The rate and equilibrium constants are temperature dependent. These expressions for silica polymorphs are obtained from literature and the forms used in this study are given in Table I. 
TABLE II. Error between analytical scaled PSD and numerical simulations at different discretizations.

\begin{tabular}{rlc}
\hline \hline$N_{F}$ & \multicolumn{1}{c}{$\Delta x$} & Error $=\sqrt{(1 / N) \sum_{i}\left(\Phi_{i}^{\text {num }}-\Phi_{i}^{\text {exact }}\right)^{2}}$ \\
\hline 200 & 0.25 & 0.1069 \\
400 & 0.125 & 0.0878 \\
800 & 0.0625 & 0.0698 \\
4000 & 0.0125 & 0.0397 \\
\hline \hline
\end{tabular}

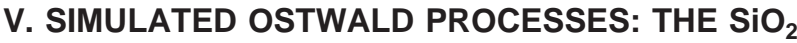 SYSTEM}

\section{A. Numerical simulations}

Ostwald processes in the multiple polymorph $\mathrm{SiO}_{2}$ system were investigated for using our model and numerical algorithm. An initially amorphous silica-rich, closed system with $60 \%$ porosity filled with an aqueous $\mathrm{SiO}_{2}$ solution was allowed to evolve at either $23^{\circ} \mathrm{C}$ or $100{ }^{\circ} \mathrm{C}$ via our model. The initial amorphous silica PSD was assumed to be lognormal with a mean diameter of $0.01 \mu \mathrm{m}$. The five silica polymorphs listed in Table II were allowed to nucleate, grow and compete for the total $\mathrm{SiO}_{2}$ pool. The initial concentration $c(0)$ of $\mathrm{SiO}_{2}$ (aq) was chosen be that in equilibrium with the initial average size, amorphous silica cluster at the temperature of the run. The heterogeneous sites have radius of $50 \AA$ and the adsorption energies for all minerals are chosen to be the same, i.e., $d_{m}=a_{\mathrm{qz}}$ for all polymorphs except as noted below where special cases were simulated to illustrate how the energetics of the nucleation site can thwart the normal Ostwald stepping sequence (see Sec. V D below). For the numerical simulations, the cluster number for transition from discrete to continuous behavior is chosen as $N=100$, and the continuous range of the logarithmic cluster number axis is divided into 200 segments $\left(N_{F}=200\right)$ with $\Delta x=0.25$. With this numerical data, the cluster size ranges from monomer to $5 \times 10^{23}$-mer is simulated for the five polymorphs, and a total of 1497 equations are solved at each time step. The results of the simulations are as follows.

The simulations illustrated a richness of nucleation and Ostwald processes. The higher free energy polymorphs precipitate first [at the expense of amorphous silica through $\mathrm{SiO}_{2}(\mathrm{aq})$ dissolved from the latter] but eventually lower free energy polymorphs nucleate and grow at the expense of the higher free energy ones. The evolution of the silica polymorph volume fractions at $23{ }^{\circ} \mathrm{C}$ is seen in Fig. 1 . The amorphous silica is quickly dissolved, allowing lower free energy ones to nucleate and grow. The intermediate polymorph $\beta$-cristobalite is essentially skipped, and $\alpha$-cristobalite dominates the system at the expense of amorphous silica. But soon, $\alpha$-cristobalite also starts to dissolve, and is replaced by chalcedony and ultimately, quartz. Quartz always has higher volume fraction than chalcedony because nucleation site adsorption energy favors quartz over chalcedony. As the thermodynamic advantage of quartz over chalcedony is rather small, these two polymorphs coexist over long times. Finally, chalcedony vanishes and quartz is the only surviving phase concluding the Ostwald stepping dynamic. The variation of $\mathrm{SiO}_{2}(\mathrm{aq})$ concentration during the Ostwald stepping stage is shown in Fig. 2. The $\mathrm{SiO}_{2}(\mathrm{aq})$ concentration drops to

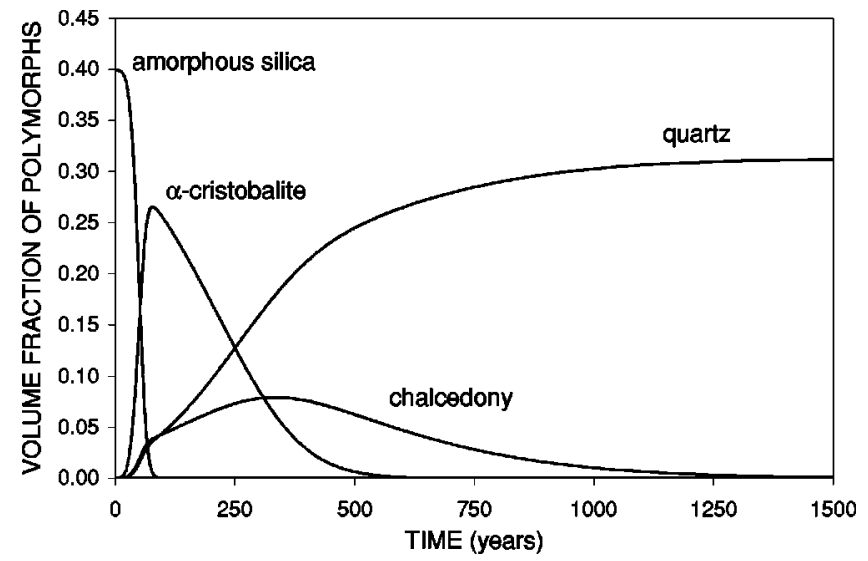

FIG. 1. Simulated silica polymorph volume fraction evolution at $23^{\circ} \mathrm{C}$. The system initially contains only amorphous silica at $40 \%$ volume fraction and finally reaches to the pure quartz state. Ostwald stepping behavior is observed; note that $\beta$-cristobalite is essentially skipped.

that of equilibrium with the dominant polymorph at each stage of the stepping.

The evolution of the quartz PSD at $23{ }^{\circ} \mathrm{C}$ is shown in Fig. 3 during the Ostwald stepping and ripening stages. The PSD is displayed as $n^{2} \xi_{n}$ vs $\log (n)$; the area under this function for any interval of $\log (n)$ is proportional to the quartz volume in that interval at that time. The long time fate of this system is that of pure quartz evolving according to Ostwald ripening, i.e., larger quartz crystals grow at the expense of the smaller ones. As our model approaches the LSW equations for large $n$, it should recover the LSW scaling behavior. This is suggested in Fig. 3 where a steady pulse of $n^{2} \xi_{n}^{\mathrm{qz}}(t)$ is seen to emerge at long times, propagating to the right with maximum located at a point that advances as $t^{1 / 2}$. This behavior, different from the classic $t^{1 / 3}$ behavior, is justified for our model via the scaling analysis presented in the next section.

A difference in low and high temperature cases is that the lower surface free energy of the intermediate phases relative to quartz is masked at higher temperatures. However, the low bulk free energy advantage of quartz is diminished with increasing temperature. The net result is that as temperature

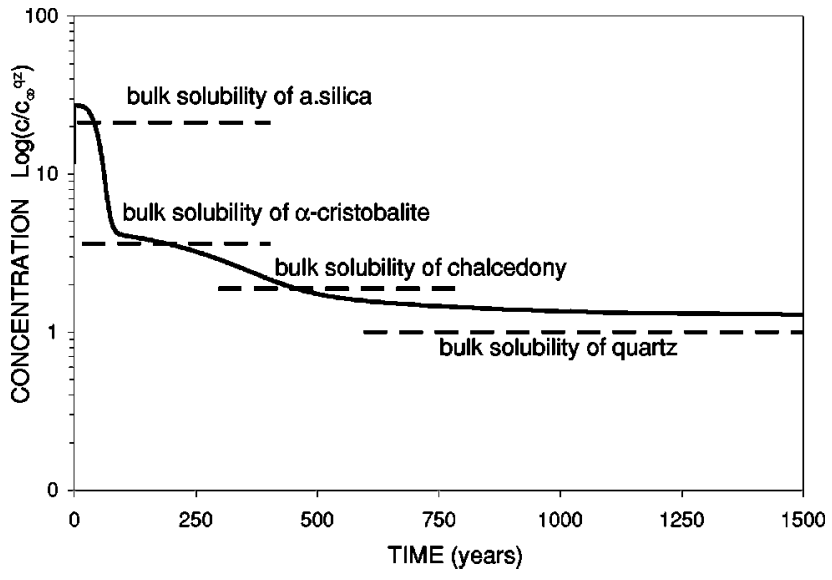

FIG. 2. The variation of $\mathrm{SiO}_{2}(\mathrm{aq})$ concentration during the Ostwald stepping for the simulation of Fig. 1 . 


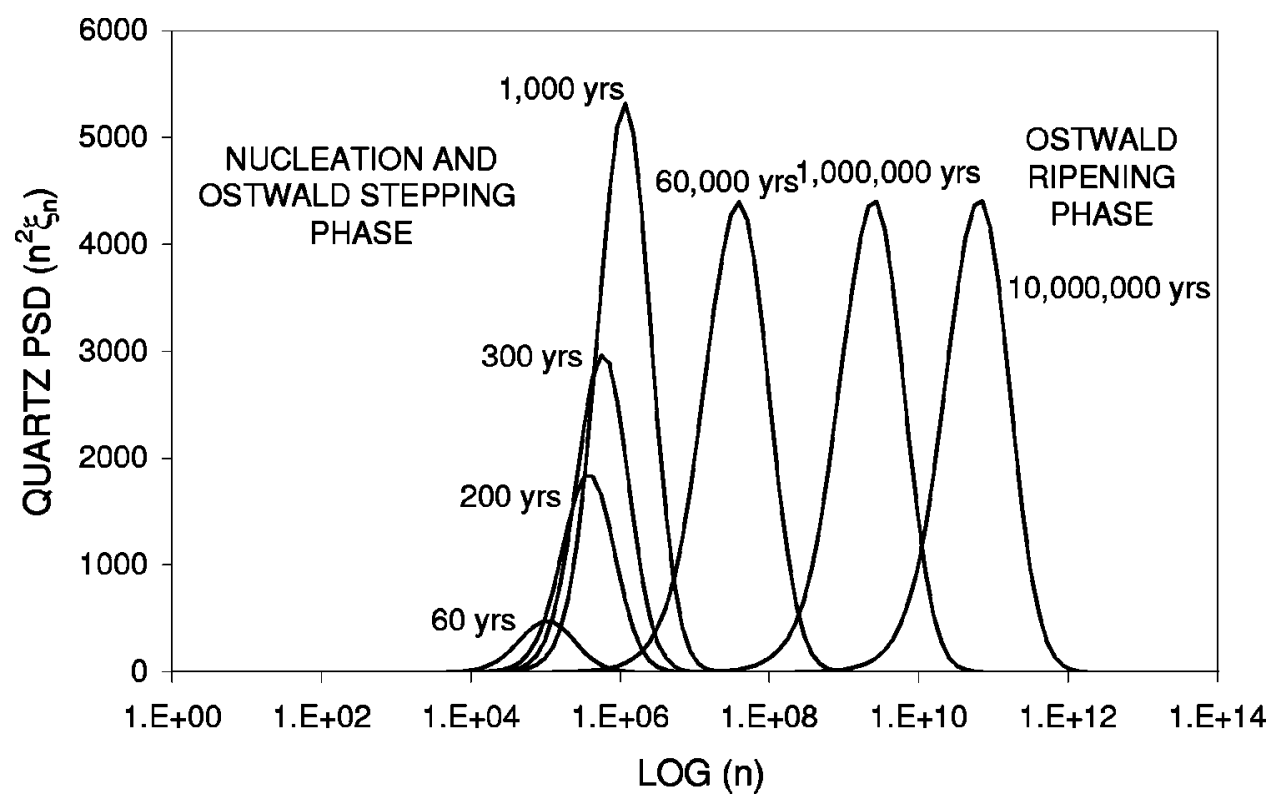

FIG. 3. Simulation evolution of the quartz PSD during the Ostwald stepping and ripening for the case of Fig. 1.

increases, the overall process amorphous silica $\rightarrow$ quartz polymorph stepping is accelerated. In the Ostwald ripening phase, when compared for the same time interval, higher temperature evolution yields larger average size for quartz than low temperature evolution. Ripening of quartz crystals at $23{ }^{\circ} \mathrm{C}$ and $100{ }^{\circ} \mathrm{C}$ are given in Fig. 4 as a logarithmic plot of average crystal size vs time. We define the average crystal size as the median diameter. As seen from this figure, the average size of quartz evolved at $100{ }^{\circ} \mathrm{C}$ is around one order of magnitude larger than that of evolved at $23{ }^{\circ} \mathrm{C}$. It is interesting that the ripening stage at both temperatures can be distinguished from the earlier stages by a linear increase of the average size with time in the logarithmic plot. The lines for different temperatures have the same slope of 0.5 , which yields a growth rate proportional to $t^{1 / 2}$, independent of the temperature of evolution. In the next section, we will justify this result via scaling analysis.

\section{B. Scaling behavior}

The scaling (long time Ostwald ripening) behavior of the PSD is a rather general property of the model of Sec. III for a large class of systems. According to Eq. (3.4) the PSD $y(n, t)(\equiv n \widetilde{\xi}(n, t))$ for a single polymorph system satisfies

$$
\frac{\partial y}{\partial t}+n \frac{\partial}{\partial n}\left[\frac{k_{n}}{n}\left(K_{n} c-1\right) y\right]=0 .
$$

In the asymptotic regime $k_{n}$ and $K_{n}$ are determined by the dependence of the surface area on $n$. For smooth clusters the surface area is proportional to $n^{2 / 3}$ but for fractal clusters it can grow with a higher power than $\frac{2}{3}$. Furthermore, $C$ may have a factor of $n^{-1 / 3}$ for diffusion controlled growth. To generalize our treatment, we introduce a phenomenological exponent $\omega$ such that

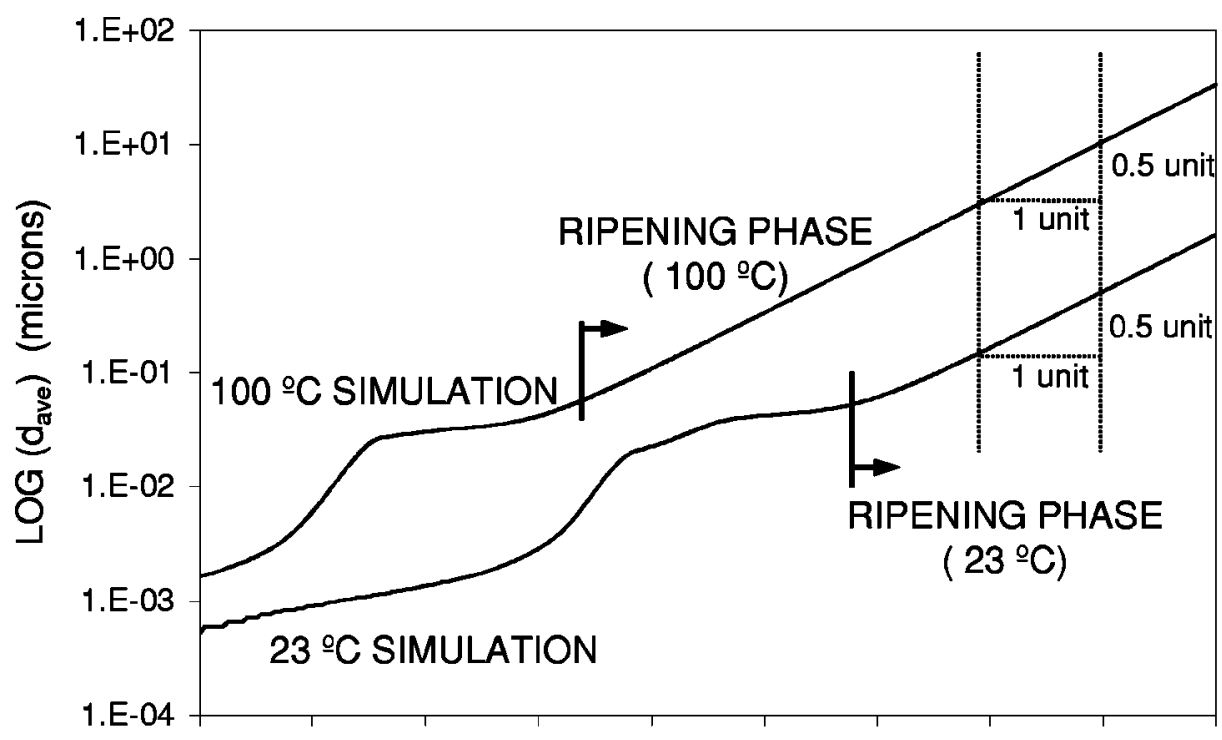

FIG. 4. Temporal evolution of the average particle size illustrating the emergence of $t^{1 / 2}$ scaling at long times, regardless of temperature.

1.E-02 1.E-01 1.E+00 1.E+01 1.E+02 1.E+03 1.E+04 1.E+05 1.E+06 1.E+07 


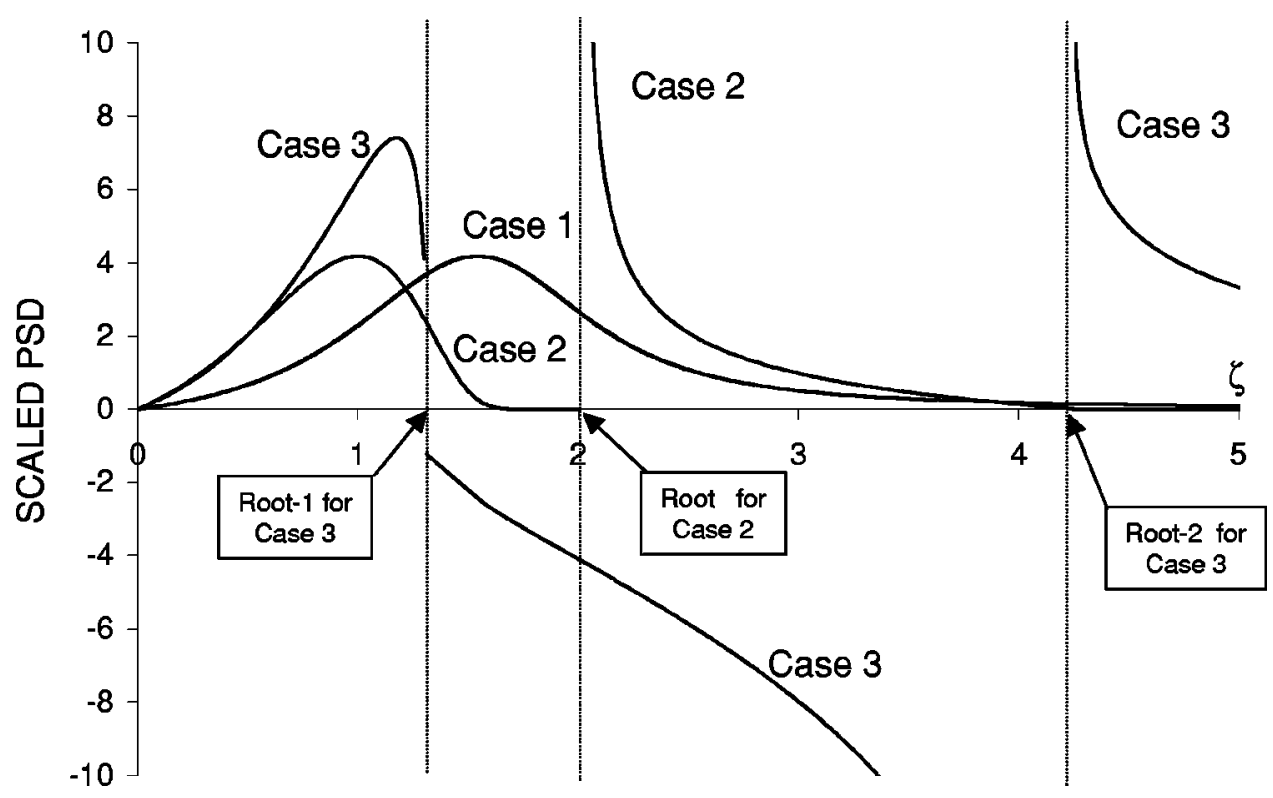

FIG. 5. Solutions for the asymptotic Eq. (5.9) at the ripening stage for different values of $\gamma$. Note that only $\gamma$ $=\frac{1}{4}$ gives a physically relevant PSD.

$$
\begin{aligned}
& K_{n} \underset{n \rightarrow \infty}{\sim} \frac{1}{c_{\infty}}\left[1-\frac{\alpha}{n^{\omega}}\right], \\
& \frac{k_{n}}{n} \underset{n \rightarrow \infty}{\sim} \frac{k}{n^{\omega}},
\end{aligned}
$$

where $\omega=1 / 3$ for the simulations of the previous section. In arriving at this ansatz we restrict our treatment to the reaction-limited case; for the diffusion-limited case $k_{n}$ should vanish as $n^{-\omega-(1 / 3)}$ and not as $n^{-\omega}$. Furthermore, we assume that the surface area increases as $n^{1-\omega}$ as $n \rightarrow \infty$. Introducing the supersaturation $\sigma$ via $c_{\infty} \sigma=c-c_{\infty}$, Eq. (5.1) becomes in the asymptotic regime,

$$
\frac{\partial y}{\partial t}+n \frac{\partial}{\partial n}\left[\frac{k}{n^{\omega}}\left(\sigma-\frac{\alpha}{n^{\omega}}\right) y\right]=0 .
$$

Let us now investigate similarity solutions of Eq. (5.4) that generalize the LSW asymptotic results for the present $\omega$-system. To do so we introduce the similarity variable $\zeta$ and a modified time $\tau$ such that

$$
\zeta=\frac{\sigma n^{\omega}}{\alpha}, \quad d \tau=\frac{k \omega}{\alpha} \sigma^{2} d t .
$$

With this we find

$$
\left(\frac{\partial y}{\partial \tau}\right)_{n}+\zeta \frac{\partial}{\partial \zeta}\left[\frac{1}{\zeta}\left(1-\frac{1}{\zeta}\right) y\right]=0 .
$$

In this equation we have made explicit the fact that the first term is a partial derivative with respect to $\tau$ at constant $n$ (not ५).

Conservation of $\mathrm{SiO}_{2}$ units during ripening yields

$$
Q=\sigma+\frac{1}{c_{\infty}} \int_{0}^{\infty} y(n, t) d n,
$$

where $Q$ has a constant, finite value determined by the initial conditions. In the asymptotic regime, $\sigma \rightarrow 0$ and this equation takes the form,

$$
Q=\frac{1}{c_{\infty} \omega} \int_{0}^{\infty} \frac{n y(n, \tau)}{\zeta} d \zeta,
$$

which suggests that as $Q$ is constant, there may be solutions such that $n y(n, t)$ might be a function of the similarity variable $\zeta$ only. Defining $Y(\zeta)=n y(n, t) / \zeta^{1+(1 / \omega)}$ and substituting into Eq. (5.6), the modified PSD function $Y$ satisfies

$$
-Y+\frac{d}{d \zeta}[\omega u(\zeta, \gamma) Y]=0,
$$

where

$$
u(\zeta, \gamma)=-\frac{1}{\zeta}\left[\zeta^{2}-\frac{1}{\gamma} \zeta+\frac{1}{\gamma}\right],
$$

and $\gamma$ is defined via

$$
\gamma=-\frac{1}{\sigma} \frac{d \sigma}{d \tau}=-\frac{\alpha}{k \omega} \frac{1}{\sigma^{3}} \frac{d \sigma}{d t} .
$$

If $Y$ is to be a similarity solution (i.e., $Y$ is a function of $\zeta$ but not of $\tau$ ), then $\gamma$ must be a constant. The mass conservation equation with the new variables has the form

$$
Q=\frac{1}{\omega c_{\infty}} \int_{0}^{\infty} \zeta^{1 / \omega} Y(\zeta) d \zeta .
$$

We are thus seeking a solution of Eq. (5.9) which satisfies the normalization condition (5.12). The solution of Eq. (5.9) is

$$
Y(\zeta)=\frac{A}{u(\zeta, \gamma)} e^{(1 / \omega) \int_{0}^{\zeta} 1 / u(\zeta, \gamma) d \zeta} .
$$


This equation has three types of solution depending on the value of $\gamma$. These solutions correspond to the cases where $u$ has no root, one double root and two distinct roots. We examine each case separately to determine the value of $\gamma$ for which the solution (5.13) is physically relevant.

Case 1. No real roots: $\Delta=1 / \gamma^{2}-4 / \gamma<0$,

$$
\begin{aligned}
Y(\zeta)= & A_{1} \frac{\zeta}{\left(\zeta^{2}-(1 / \gamma) \zeta+1 / \gamma\right)^{1+(1 / 2 \omega)}} \\
& \times e^{\{(1 / \omega \gamma \sqrt{-\Delta}) \arctan [2 \zeta(1 / \gamma) / \sqrt{-\Delta}]\}} .
\end{aligned}
$$

Case 2. One double root: $\zeta_{1}=\zeta_{2}=2(\gamma=1 / 4)$,

$$
Y(\zeta)=A_{2} \frac{\zeta}{\left(\zeta^{2}-4 \zeta+4\right)^{1+(1 / 2 \omega)}} e^{[2 / \omega(\zeta-2)]}
$$

Case 3. Two distinct roots: $\zeta_{1,2}=1 / 2 \gamma \pm(1 / 2) \sqrt{\Delta}$, and $\Delta$ $=1 / \gamma^{2}-4 / \gamma>0$,

$$
Y(\zeta)=\left\{\begin{array}{cc}
A_{3} \frac{\zeta}{\left(\zeta_{1}-\zeta\right)^{1+(1 / 2 \omega)-(1 / 2 \gamma \omega \sqrt{\Delta})}\left(\zeta_{2}-\zeta\right)^{1+(1 / 2 \omega)+(1 / 2 \gamma \omega \sqrt{\Delta})},} & \zeta<\zeta_{1} \\
-A_{4} \frac{\zeta}{\left(\zeta-\zeta_{1}\right)^{1+(1 / 2 \omega)-(1 / 2 \gamma \omega \sqrt{\Delta})}\left(\zeta_{2}-\zeta\right)^{1+(1 / 2 \omega)+(1 / 2 \gamma \omega \sqrt{\Delta})},} & \zeta_{1}<\zeta<\zeta_{2} \\
A_{5} \frac{\zeta}{\left(\zeta-\zeta_{1}\right)^{1+(1 / 2 \omega)-(1 / 2 \gamma \omega \sqrt{\Delta})}\left(\zeta-\zeta_{2}\right)^{1+(1 / 2 \omega)+(1 / 2 \gamma \omega \sqrt{\Delta})},} & \zeta>\zeta_{2}
\end{array}\right.
$$

for constants $A_{1}, \ldots, A_{5}$ to be determined. In Fig. 5, these three cases are plotted. In all cases independent of $\omega$, the large $\zeta$ behavior of the integrand in Eq. (5.12) is $\zeta^{1 / \omega} Y(\zeta)$ $\rightarrow{ }_{\text {large } \zeta} 1 / \zeta$; therefore the large $\zeta$ contribution to the integral appears to be logarithmically divergent. Therefore, $Y(\zeta)$ should have an augmented form: it is one of the solutions given in Eq. (5.14) between $0 \leqslant \zeta \leqslant \zeta^{*}$ and it must be equal to zero after the point $\zeta=\zeta^{*}$.

The key to the divergency dilemma is basically an argument of causality. If at time zero, there were only finite size particles, then in any finite time the PSD must be identically zero for all $\zeta$ beyond a cutoff value $\zeta^{*}$.

The $D$-term in Eq. (3.4) involves, in the present notation, a term of the form $d^{2} Y / d \zeta^{2}$ if these correction terms were retained. Thus, $d Y / d \zeta$ must be continuous for all $\zeta$; otherwise the $D$-term would be infinite and the above solutions would be unphysical as the $D$-terms were assumed to be small. By a similar argument, the transformation of the discrete chemical kinetic model to the continuous one yields a sum of terms involving all derivatives. Thus, if any derivative of $Y$ with respect to $\zeta$ is discontinuous at $\zeta=\zeta^{*}$, then the solution will also be unphysical. More dramatically, these types of lack of smoothness in $Y$ would imply a breakdown of the entire continuum theory, a physically nonintuitive result. Thus, this implies, $Y$ and all of its derivatives must be zero at $\zeta=\zeta^{*}$.

For the Case $1\left(\gamma>\frac{1}{4}\right), Y$ has no zero for $0<\zeta<\infty$. In Case $3\left(\gamma<\frac{1}{4}\right)$, the solution has a divergence to the left of where it has its first zero so that it cannot be a physical solution if $\gamma<\omega(4 \omega+2) /(4 \omega+I)^{2}=\gamma^{*}$. For $\gamma^{*}<\gamma<\frac{1}{4}$, a surprising situation arises: there is a one parameter family of scaling solutions for which $Y$ and its first derivative is zero at the first root, $\zeta_{1}$. For a given $\omega$, with $\gamma$ in this acceptable range, the mode particle sizes of these solutions have the same scaling exponent of time (see below). We do not investigate further here which of these solutions is the most stable. However, as noted above all derivatives of $Y$ with respect to $\zeta$ must vanish at $\zeta^{*}$. This is the only case for $\gamma=\frac{1}{4}$, Case 2 . For Case $2\left(\gamma=\frac{1}{4}\right), Y$ and all its derivatives vanish at $\zeta=2$. Note that for this case $\gamma=\frac{1}{4}$ and from Eq. (5.10) we see that $u$ and $d u / d \zeta$ vanish at $\zeta=2$ where $Y$ vanishes. Thus, we find $\gamma=\frac{1}{4}$ and $\zeta^{*}=2$. With this the asymptotic PSD is given by

$Y(\zeta)=\left\{\begin{array}{c}A \zeta^{*(1 / \omega)} e^{(1 / \omega)} \frac{\zeta}{\left(\zeta^{*}-\zeta\right)^{2+(1 / \omega)}} e^{-(1 / \omega)\left[\zeta^{*} /\left(\zeta^{*}-\zeta\right)\right]} \\ \text { for } \zeta \leqslant \zeta^{*}=2 \\ 0, \quad \text { for } \zeta \geqslant \zeta^{*}\end{array}\right.$.

The constant $A$ is obtained from the mass conservation condition (5.12) one finds

$$
A=\frac{Q c_{\infty} \omega}{\int_{0}^{\zeta^{*}} \zeta^{* 1 / \omega} e^{1 / \omega} \frac{\zeta^{1+(1 / \omega)}}{\left(\zeta^{*}-\zeta\right)^{2+(1 / \omega)}} e^{-(1 / \omega)\left[\zeta^{*} /\left(\zeta^{*}-\zeta\right)\right]} d \zeta} .
$$

In conclusion, $\gamma$ is determined as an eigenvalue and $A$ is implied by normalization. The cutoff at $\zeta^{*}=2$ is a property of the form of the PSD equation and is required by the causality. The vanishing of all derivatives at $\zeta=\zeta^{*}$ and the continuity of $d Y / d \zeta$ for $0 \leqslant \zeta \leqslant \zeta^{*}$ shows the self-consistency of our continuum approximation to the discrete chemical kinetics model.

For rough (fractal) particles, $\omega<\frac{1}{3}$. Three cases for $\omega$ $<\frac{1}{3}$ are compared with the smooth particle case in Fig. 6 . The PSD's are scaled to have unit area under the curves. The maximum point of the PSD's are at $\zeta^{*} / \sqrt{1+(1 / \omega)}$, and the 


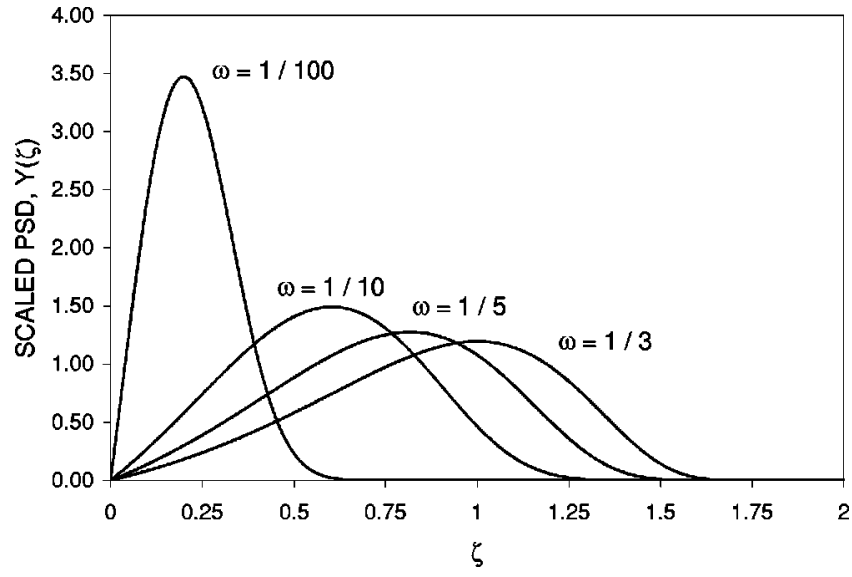

FIG. 6. Comparison of the PSD, $Y(\zeta)$, for smooth particles $(\omega=1 / 3)$ and for rougher (fractal-like) cases $\omega<1 / 3$.

location of maximum points moves towards the left as $\omega$ decreases, yielding narrower PSD's for rougher particles than for smoother ones.

Our numerical results corresponds to $\omega=1 / 3$, in which the surface area is proportional to $n^{2 / 3}$. For this case $A$ $\cong 1.05 Q c_{\infty}$. As seen from Fig. 6 , at $\zeta=1, Y(\zeta)$ has a maximum. Therefore from the definition of the variable $\zeta$, $\left(n^{1 / 3}\right)_{\max }=\alpha / \sigma$, where $\left(n^{1 / 3}\right)_{\max }$ represents the value of $n^{1 / 3}$ at which the asymptotic PSD $Y(\zeta)$ has a maximum.

Since $\gamma=1 / 4$, Eq. (5.11) implies

$$
\gamma=\frac{1}{4}=-\frac{3 \alpha}{k \sigma^{3}} \frac{d \sigma}{d t},
$$

which implies that

$$
\left(\frac{1}{\sigma}\right)^{2}=\frac{1}{\alpha^{2}}\left[\left(n^{1 / 3}\right)_{\max }\right]^{2}=\frac{k}{6 \alpha} t+\text { constant }, \quad \omega=\frac{1}{3} .
$$

This determines the variations of concentration and maximum of the PSD with time in the asymptotic regime. Since $n^{1 / 3}$ is a measure of crystal diameter for this smooth cluster case, we can conclude that, in surface kinetic controlled growth systems, the square of average (mode) particle diameter grows linearly with time. In the LSW theory, where growth is diffusion controlled, the cube of the average diameter grows linearly with time. Hence, kinetic limited ripening is faster as one expects.

Ripening for fractal clusters is expected to be faster than for smooth ones. From the definition of $\zeta$, the maximum of the PSD is at

$$
\zeta_{\max }(\omega)=\frac{\sigma(t)\left(n^{\omega}\right)_{\max }}{\alpha} .
$$

Using Eq. (5.11) for the $t$-dependence of $\sigma$ and noting that

$$
\zeta_{\max }(\omega)=\frac{2}{\sqrt{1+(1 / \omega)}},
$$

one finds that

$$
\left(n^{1 / 3}\right)_{\max }=\left(\frac{2 \alpha k \omega^{2}}{1+\omega} k\right)^{1 / 6 \omega} t^{1 / 6 \omega} .
$$

This agrees with Eq. (5.18) for $\omega=\frac{1}{3}$ and shows that ripening accelerates as the roughness of the clusters increases (i.e., as $\omega$ decreases).

\section{Comparison of asymptotic and numerical results}

In the numerical simulation, we obtain $y(n, t)$ at various times. To test how accurate our numerical scheme is, we investigate the following:

(1) Do the numerical results yield a time independent scaled form?

(2) If there is a numerical time-independent PSD, does it converge to the analytical scaled PSD and what is the convergence rate?

(3) Is there a numerical power law relating average (or modal) diameter to time and is it converging to the analytical square law?

For the purpose of comparison, a pure quartz system with initially having the average diameter of 0.01 microns

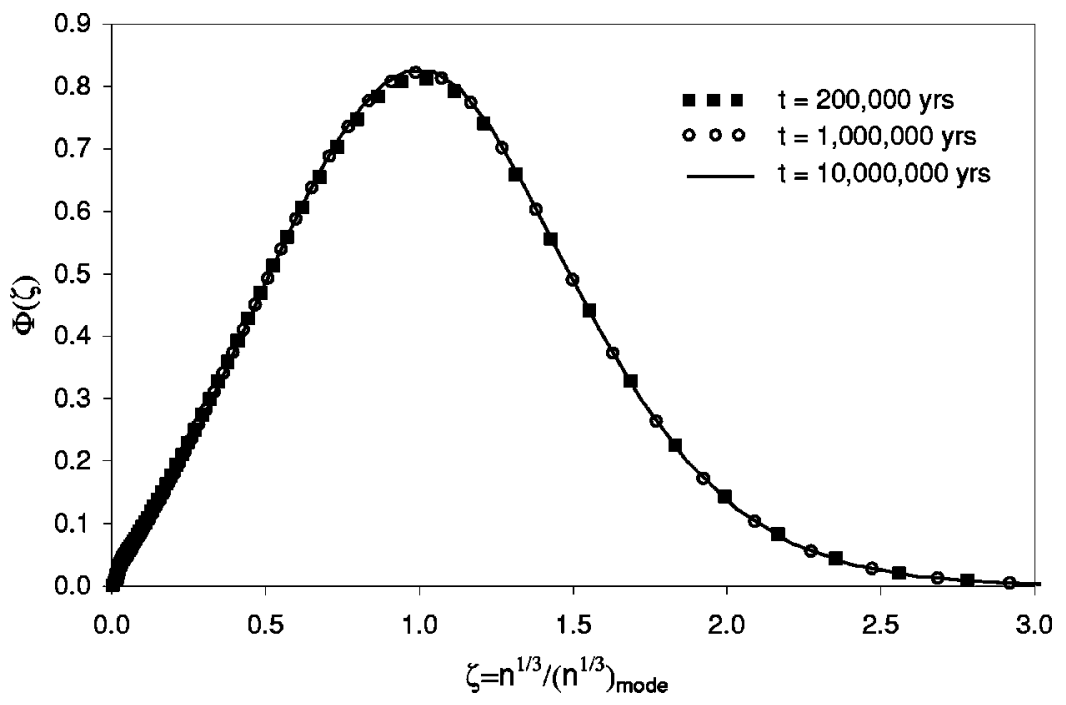

FIG. 7. The numerical algorithm yields a time independent scaled PSD. The scaled PSD's of the numerical simulations at 200000,1 million, and 10 million years exactly match each other. Note that the $x$-axis essentially particle diameter scaled with the average (mode) diameter. 

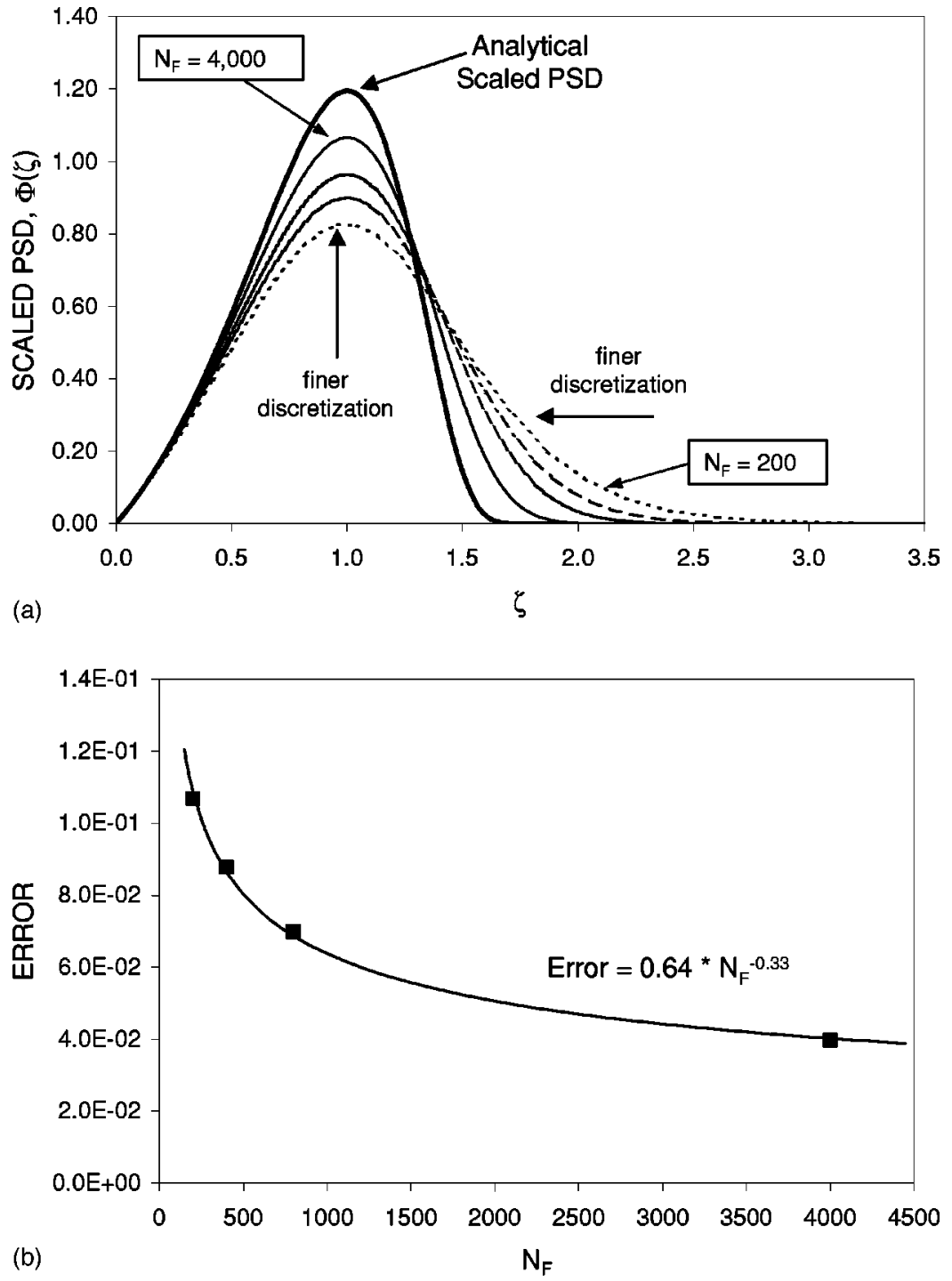

FIG. 8. Convergence of numerical results to the analytical solution, (a) comparison of numerical scaled PSD with the analytical one for $N_{F}=200,400,800$, and 4000. Note that as $N_{F}$ increases, the discretization becomes finer, and the numerical results approach to the analytical one, (b) the error of numerical results versus the number of discretization elements $N_{F}$. and volume fraction of 0.40 was investigated at $23^{\circ} \mathrm{C}$. The numerical simulations are done with $N=100$ and with $N_{F}$ varying from 200 to 4000 . At each time step, the numerical simulation gives the quartz PSD in the form of $\xi_{n}^{\mathrm{qz}}$ in the discrete range $(1 \leqslant n \leqslant N)$ and $y^{\mathrm{qz}}(n, t)$ in the continuous range. From the definitions given in the previous section, the scaled PSD, $Y$, will be related to $y(n, t)$ via

$$
Y=\frac{\alpha^{4}}{(\sigma(t))^{4}} \frac{y(n, t)}{n^{1 / 3}} .
$$

Therefore, for any given time, the scaled PSD is proportional to $\Phi^{\mathrm{qz}}(n)=y^{\mathrm{qz}}(n) / n^{1 / 3}$ of the numerical results, and $\left(n^{1 / 3}\right)_{\max }^{\mathrm{qz}}$ can easily be evaluated from the position of the maximum value of $\Phi^{\mathrm{qz}}$. If there is a time-independent scaled PSD, the numerical results illustrated in the form of $\Phi^{\mathrm{qz}} \mathrm{vs}$ $n^{1 / 3} /\left(n^{1 / 3}\right)_{\max }^{\mathrm{qz}}$ will coincide at every timestep. In Fig. 7 , the numerically scaled PSD, $\Phi^{\mathrm{qz}}$, is plotted at 200000,1 million, and 10 million years of simulation with $N=100$ and $N_{F}=200$. As seen from this figure, the scaled PSD at 200000 and 1 million years (dotted curves) fit almost ex- actly to the PSD at 10 million years (solid curve). This implies that the numerical simulation yields a time-independent scaled PSD.

The convergence of the numerical scaled PSD's to the analytical one is shown in Fig. 8(a). The numerical PSD's correspond to the simulations with $N_{F}=200,400,800$, and 4000. The numerical scaled PSD's are more dispersed than the analytical one, but converge to the analytical PSD as the discretization of the numerical scheme gets finer. The dispersed shape of numerical PSD has two origins. First, for the analytical solution, we ignored the $D$-term of Eq. (3.4). The $D$-term widens the PSD via a diffusionlike effect, especially at the early stages of ripening (when the typical values of $n$ are not so large). The deviation of the numerical solutions from the analytical asymptotic one due to $D$-term is independent of numerical discretization, and vanishes as the system ripens. The second reason for the dispersion of the numerical results is the upwinding scheme used in the solution of the continuous part. ${ }^{20}$ By applying the upwinding scheme, the oscillations due to the transport errors in the numerical solution of the advective-type equation (3.4) are overcome at the cost of an effective numerical dispersion. The error due to 


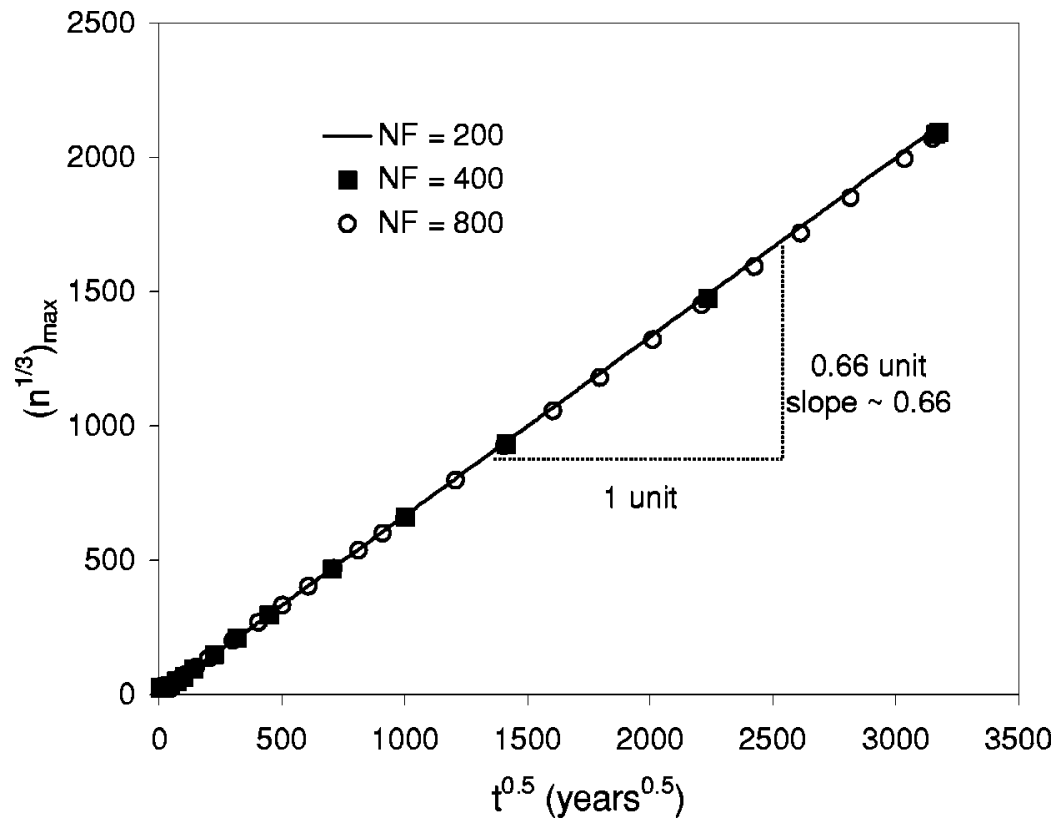

FIG. 9. Linear variation between $\left(n^{1 / 3}\right)_{\max }$ and $\sqrt{t}$ is obtained in numerical simulations. The solid line is the numerical evolution with $N_{F}=200$, and the dotted ones correspond to the finer discretizations $\left(N_{F}=400\right.$ and 800).

the upwind differencing depends on the discretization, and becomes smaller as the discretization gets finer. The errors for the numerical simulations are listed for different discretizations in Table II and plotted in Fig. 8(b). The error is calculated as the square root of the sum of the square of differences at each discretization point. By curve fitting these values, we obtain error as a function of the number $N_{F}$ of discretization elements, we find

$$
\text { Error }=0.64\left(\frac{1}{N_{F}}\right)^{0.33} \text {. }
$$

The slowness of the convergence is likely associated with the simple constant finite element functions used. However, these elements had the convenient property that total mass was exactly conserved. Finally, this extrapolation formula may be generalized and used to improve numerical simulations to infinitely fine mesh results. With this we conclude that our numerical approach yields well controlled approximations to the PSD problem. ${ }^{21,22}$

In Fig. 9, $\left(n^{1 / 3}\right)_{\max }$ vs $\sqrt{t}$ is plotted for numerical simulations with $N_{F}=200,400$, and 800. As stated before, $\left(n^{1 / 3}\right)_{\max }$ represent the value of $n^{1 / 3}$ at which the asymptotic PSD $Y(\zeta)$ has a maximum and it is a measure of the average diameter for smooth particles. From this figure, it is seen that $\left(n^{1 / 3}\right)_{\max }$ is linearly proportional to the square root of time. Therefore, we can conclude that there is a numerical power law relating average (or modal) diameter to time. The proportionality constants (the slope of the growth curves) obtained from the numerical simulation results are 0.666 , 0.659 , and 0.656 for $N_{F}=200,400$, and 800 , respectively. The sensitivity of these slopes to the numerical discretization is much less than that of the PSD curves. The exact proportionality constant of the growth relation was given in Eq. (5.18). Rearranging this equation yields the analytical relation between $\left(n^{1 / 3}\right)_{\max }$ and $\sqrt{t}$ as

$$
\left(n^{1 / 3}\right)_{\max }=\sqrt{\frac{k \alpha}{6}} t^{1 / 2}+\text { constant. }
$$

Using the data given in Appendix $\mathrm{B}$ with the definitions in Sec. V B, for quartz at $23{ }^{\circ} \mathrm{C}$,

$$
\begin{aligned}
& k=\bar{k}_{\mathrm{qz}} \phi^{3} \sqrt{36 \pi}\left(\frac{\bar{V}_{\mathrm{qz}}}{N^{0}}\right)^{2 / 3}=0.21138\left[\text { year }^{-1},\right. \\
& \alpha=\frac{2}{3 R T} b_{\mathrm{qz}}=12.104,
\end{aligned}
$$

the analytical proportionality constant becomes $\sqrt{k \alpha / 6}$ $=0.653$. Unlike the PSD errors listed in Table II, the numerical error of growing rate in the $N_{F}=200$ simulation is less than $2 \%$, and it decreases rapidly as the mesh gets finer.

\section{Nucleation site energetics and Ostwald stepping}

In the numerical simulations of Sec. V A, the adsorption energies for all polymorphs are assumed to be the same. The effect of variation in adsorption energy to promote the initiation of one of the polymorphs is seen in the simulation of Fig. 10(a). There a case is run wherein all data are as in the previous runs of Sec. V A, except that the adsorption energy of $\beta$-cristobalite was taken to have the lower value of $2 a_{\mathrm{qz}}$ while the other polymorphs had the same value. Unlike for the symmetric adsorption case of Fig. 1, $\beta$-cristobalite is initiated and dominates for long times. Favoring $\beta$-cristobalite suppress $\alpha$-cristobalite initiation. The change in the adsorption energies also effects the duration of the Ostwald stepping stage. For the unsymmetric adsorption case considered, Ostwald stepping takes much longer than for the symmetric case.

In Fig. 10(b), the results of another simulation are given for a case where the adsorption energy of quartz is higher than that of the other polymorphs via $d_{\mathrm{qz}}=0.85 a^{\mathrm{qz}}$. As a result, chalcedony dominates over quartz for a long time; but 

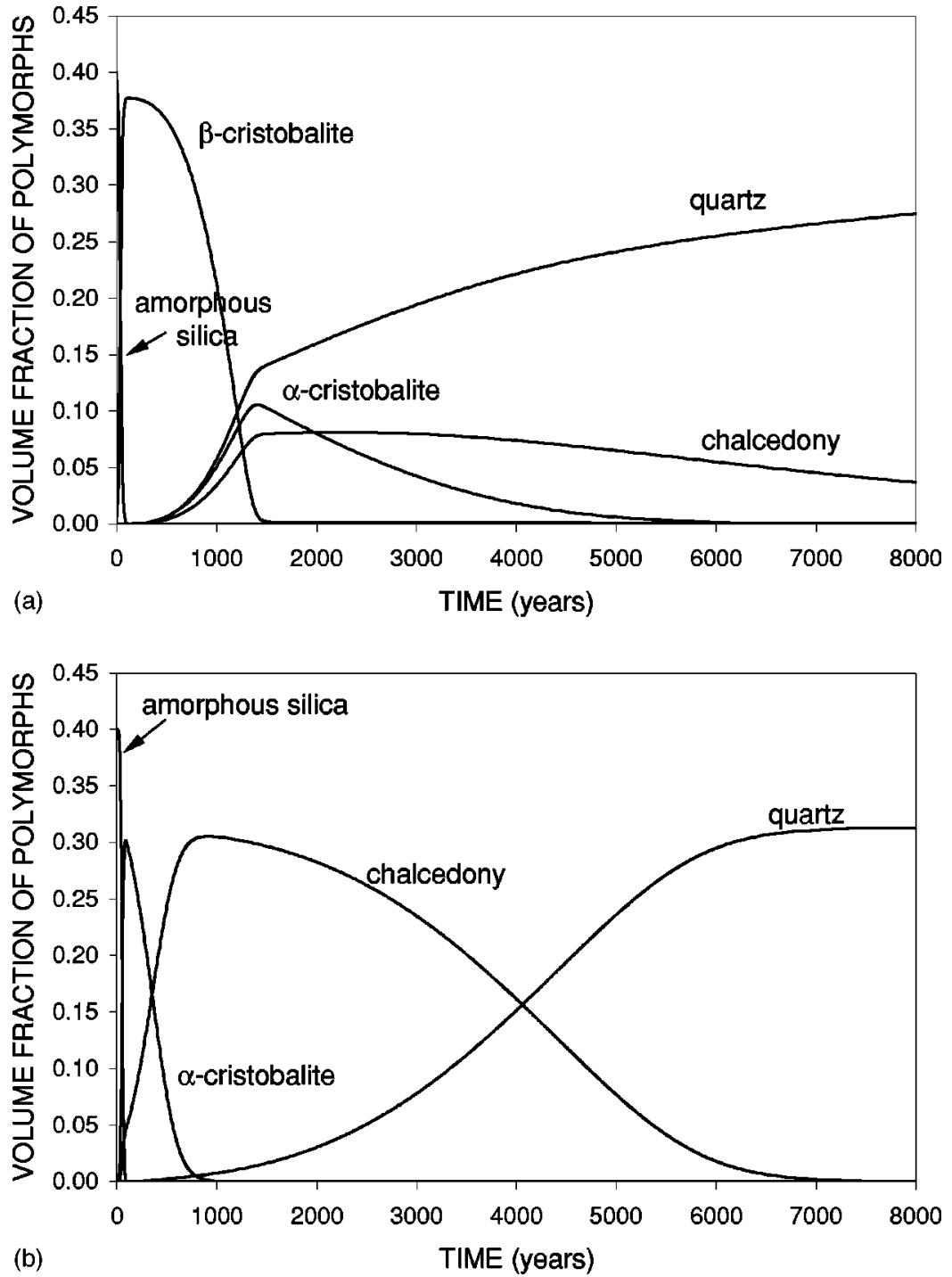

FIG. 10. The effect of nucleation site energy on the Ostwald stepping scenario, simulations at $23{ }^{\circ} \mathrm{C}$ are repeated with the unsymmetrical adsorption energies yielding, (a) $\beta$-cristobalite favored initiation $\left(d_{\beta-\mathrm{cr}}\right.$ $=2 a^{\mathrm{qz}}$, and $d_{m}=a^{\mathrm{qz}}$ for all other polymorphs), (b) quartz unfavored initiation $\left(d_{\mathrm{qz}}=0.85 a^{\mathrm{qz}}\right.$, and $d_{m}=a^{\mathrm{qz}}$ for all other polymorphs). Note the persistence of chalcedony in case (b). Compare these results to that of Fig. 1 ; note the difference in time scale. eventually, the system reaches the pure quartz state. A further increase in the quartz adsorption energy results in a longer time for the system to reach the pure quartz state. Although the system will always end up at the lowest energy polymorph, depending on the initiation scenario, the time required to achieve it can be so long that it may become impossible to obtain quartz even on a geological time scale. Thus, the microstructure of the adsorption site can dramatically change the Ostwald stepping scenario.

\section{CONCLUSIONS}

We have presented a viable approach for simulating the microscopic (adsorption and nucleation) stage to the macroscopic particle growth stage of precipitation. The boundary condition on our extended LSW equation provides an essentially seamless integration of the chemical kinetic and continuous formulations.

The four stages of precipitation can each profoundly affect the evolution of the system. Our conclusions are based on a new treatment of the chemical kinetic model of precipitation that captures the evolution of clusters on over 23 or- ders of magnitude in size, providing an integration of the micro-, meso-, and macroscopic scales.

The first stage, adsorption at a heterogeneous site, provides a way to, in part, avoid the free energy barrier presented by surface free energy. It also serves to introduce lower free energy phases early on in the overall process, allowing the system to skip some polymorphs or other phases in the Ostwald stepping sequence. The energetics of the adsorption site can be so favorable so an intermediate energy polymorph that lower free energy polymorphs can be blocked for exceedingly long times. Similarly, if lower free energy polymorphs are energetically strongly unfavored at the nucleation site, they can be inhibited from forming.

The nucleation stage is dominated by surface free energy effects arising from the interaction of small clusters with the phase form which it would, from the point of view of macroscopic (bulk) free energy, like to grow. Nucleation barriers are seen to be higher for lower bulk free energy phases as the latter appear to have higher surface energy. The surface free energy barriers are thwarted by higher temperature or when the spatial range of influence of the heterogeneous site exceeds the critical nucleus size. While not considered here, it 


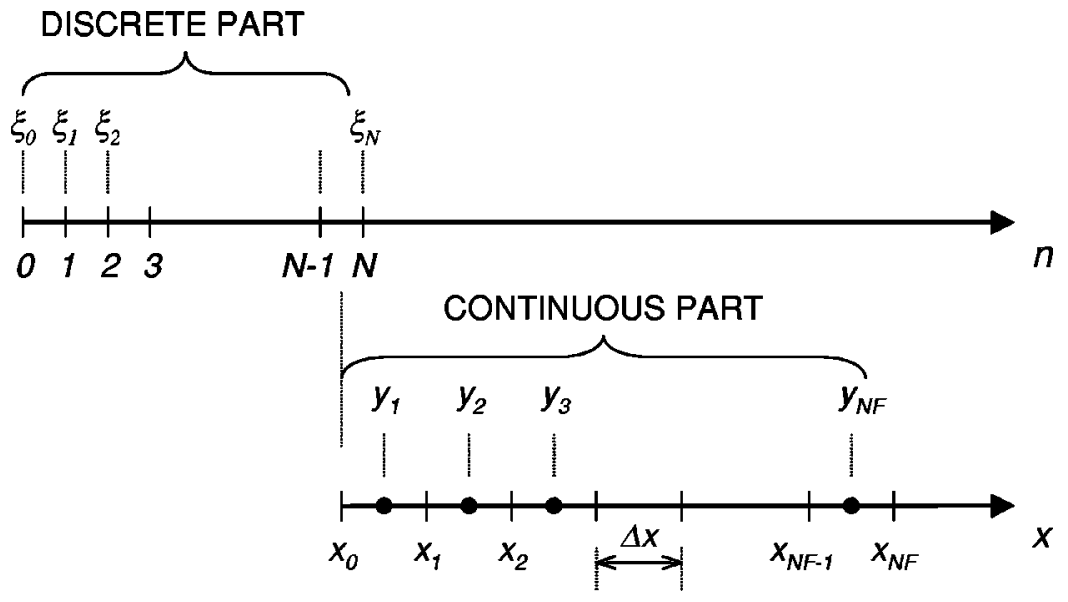

FIG. 11. Schematic presentation of the cluster size domain. The domain is divided into two parts; for small clusters $(n<N)$, discrete kinetics equations are applied, while for the large clusters, continuous kinetics equation is written with respect to the $x=\log (n)$ variable. The continuous domain is divided into equal sized elements for computational purposes in each of which the PSD, $y$, is assumed to be constant. is clear that differences in adsorption components in the growth medium with the surface of the various polymorphs can also dramatically change the order in which the various polymorphs nucleate.

The Ostwald stepping stage, the competition of the various phases for common building blocks [here $\mathrm{SiO}_{2}(\mathrm{aq})$ ] always results in the final stage with the low free energy phase. As the difference in the bulk free energies of the latter decreases, the persistence of near lowest bulk free energy phases increases. Both temperature and nucleation site energetics can dramatically change the stepping scenario.

The ripening stage, which may overlap with stepping, leads to the long time state of fewer but larger particles. At long times LSW-type scaling is achieved but with a time scaling that depends on both the rate limiting growth process (attachment kinetics versus diffusion) as well as the roughness of the particles. For smooth particles, ripening is slower than for rough (fractal) particles.

The methods used in this study can be generalized to multimineralic systems or to more complex (multicomponent) growth media. These generalizations and the extension to open and spatially nonuniform systems shall enable us to analyze the effects of the infiltration of fluids into seafloor sediments and metasomatic, weathering and many other geochemical systems as well as engineering contexts where precipitation plays an important role. In all such contexts, the methodology presented should provide an effective framework for simulating the microscopic $\rightarrow$ macroscopic transition underlying precipitation dynamics.

\section{ACKNOWLEDGMENT}

We thank the Division of Earth Sciences of Directorate for Geosciences of National Science Foundation for support of this project.

\section{APPENDIX A: DERIVATION OF THE CONTINUOUS PSD DYNAMICS}

To analyze Ostwald phenomena, we must simulate a large range of the cluster sizes. In our approach, we divided the cluster size axis into two parts at a cluster size $N$ (as seen in Fig. 11) and apply the discrete chemical kinetic formulation for the small clusters $(n<N)$. For the other part of the domain, the PSD equations are reformulated in a continuous form by defining a PSD function $\tilde{\xi}^{m}$ and $y^{m}$ which are assumed to be continuous on the cluster size axis. Starting from the definition of the continuous site fractions [Eq. (3.1)], and changing the interaction limits as

$$
\xi_{n}^{m}(t)=\int_{-1 / 2}^{1 / 2} \tilde{\xi}^{m}(n+\alpha, t) d \alpha
$$

we can apply Taylor series expansion around $n$ to obtain the explicit relation between the discrete and continuous site fractions. The integral in Eq. (A1) then becomes

$$
\begin{aligned}
\xi_{n}^{m}(t)= & \int_{-1 / 2}^{1 / 2}\left(\widetilde{\xi}^{m}(n, t)+\alpha \frac{\partial \widetilde{\xi}^{m}}{\partial n}(n, t)\right. \\
& \left.+\frac{\alpha^{2}}{2} \frac{\partial^{2} \widetilde{\xi}^{m}}{\partial n^{2}}(n, t)+\cdots\right) d \alpha .
\end{aligned}
$$

Evaluating the integral, we obtain

$$
\xi_{n}^{m}(t)=\widetilde{\xi}^{m}(n, t)+\sum_{k=1}^{\infty} \frac{2}{(2 k+I) !}\left(\frac{1}{2}\right)^{2 k+1} \frac{\partial^{2 k} \widetilde{\xi}^{m}}{\partial n^{2 k}}(n, t) .
$$

The second term is seen to be a rapidly convergent series due to the $\left[(2 k+I) ! 2^{2 k+1}\right]^{-1}$ factor (the first being 1/24). Furthermore, for large $n$, the PSD is slowly varying with respect to $n$; therefore we can neglect the second and higher order derivatives, yielding

$$
\xi_{n}^{m}(t) \cong \tilde{\xi}^{m}(n, t)
$$

Note that the assumed smoothness of the PSD, or more specifically the existence of all derivatives of the PSD with respect to $n$, has strong implications for the acceptable solutions of the continuous PSD equations (see Sec. V B).

To obtain the continuous form of the PSD equations, we insert the approximation (A4) into Eq. (2.3). From the central value theorem, the right-hand side of Eq. (2.3) may be expressed as a derivative of the continuous function $W^{m}$ at the point $n^{*}$ in the interval between $n-1$ and $n$, such that $W_{n-1}^{m}-W_{n}^{m}=-\partial W^{m} /\left.\partial n\right|_{n *}, n-1 \leqslant n^{*} \leqslant n$. Then, the discrete kinetic Eq. (2.3) takes the form, 


$$
\begin{aligned}
\frac{\partial \widetilde{\xi}^{m}(n, t)}{\partial t} & =-\left.\frac{\partial W^{m}}{\partial n}\right|_{n *} \\
& =-\frac{\partial}{\partial n}\left[k_{n}^{m}\left(K_{n}^{m} c \widetilde{\xi}^{m}(n, t)-\widetilde{\xi}^{m}(n+1, t)\right)\right]_{n *} .
\end{aligned}
$$

Using a first order Taylor expansion of $\widetilde{\xi}^{m}$ around $n$, with $n-n^{*}=\Delta$, yields

$$
\begin{aligned}
\frac{\partial \tilde{\xi}^{m}}{\partial t}= & -\frac{\partial}{\partial n}\left[k _ { n - \Delta } ^ { m } \left(\left(K_{n-\Delta}^{m} c-I\right) \tilde{\xi}^{m}\right.\right. \\
& \left.\left.-\left(K_{n-\Delta}^{m} c \Delta+(1-\Delta)\right) \frac{\partial \widetilde{\xi}^{m}}{\partial n}\right)\right] .
\end{aligned}
$$

The value of $\Delta$ is chosen in our approach to insure that the summation/integration of the site fractions over the whole range of cluster size should be unity at all times. Therefore, the time derivative of site fractions adds up to zero over full range of $n$,

$$
\frac{d \xi_{0}}{d t}+\sum_{m=1}^{M} \sum_{n=1}^{\infty} \frac{d \xi_{n}^{m}}{d t}=0
$$

where all the variables are the same as defined in Sec. II. Applying the discrete formulation up to cluster size $N-1$ and the continuous formulation for the region $N-\frac{1}{2} \leqslant n<\infty$ (as suggested in Fig. 11), the conservation of nucleation sites takes the form

$$
\frac{d \xi_{0}}{d t}+\sum_{m=1}^{M}\left\{\sum_{n=1}^{N-1} \frac{d \xi_{n}^{m}}{d t}+\int_{n-1 / 2}^{\infty} \frac{\partial \tilde{\xi}^{m}}{\partial t} d n\right\}=0 .
$$

Using Eqs. (2.3) and (A6), we obtain

$$
\begin{aligned}
\sum_{m=1}^{M} & \left\{-W_{N-1}^{m}+\left(k_{n-\Delta}^{m}\left(K_{n-\Delta}^{m} c-1\right) \tilde{\xi}^{m}(n, t)\right.\right. \\
& \left.\left.\quad-k_{n-\Delta}^{m}\left(K_{n-\Delta}^{m} c \Delta+(1-\Delta)\right) \frac{\partial \tilde{\xi}^{m}}{\partial n}(n, t)\right)_{N-1 / 2}\right\}=0 .
\end{aligned}
$$

Equation (A9) is to be satisfied for all possible cases; thus it must hold for situations in which the $\widetilde{\xi}^{m}, \xi_{n}^{m}$ are zero for all but one polymorph only. In this way, Eq. (A9) holds for every polymorph separately. Inserting the expression for $W_{n}^{m}$ given in Eq. (2.4) and evaluating the continuous part at $n$ $=N-\frac{1}{2}$, one obtains

$$
\begin{aligned}
k_{N-1}^{m}( & \left.K_{N-1}^{m} c \xi_{N-1}^{m}-\xi_{N}^{m}\right) \\
= & k_{N-(1 / 2)-\Delta}^{m}\left(\left(K_{N-(1 / 2)-\Delta}^{m} c-1\right) \widetilde{\xi}^{m}\left(N-\frac{1}{2}, t\right)\right. \\
& \left.\quad-\left(K_{N-(1 / 2)-\Delta}^{m} c \Delta+(1-\Delta)\right) \frac{\partial \widetilde{\xi}^{m}}{\partial n}\left(N-\frac{1}{2}, t\right)\right) .
\end{aligned}
$$

From Eq. (A10), it can be concluded that, $\Delta=\frac{1}{2}$, and the boundary conditions for the discrete and continuous formulations at the interface which satisfy Eq. (A10) are

$$
\begin{aligned}
& \xi_{N-1}^{m}=\left.\widetilde{\xi}^{m}\right|_{n=N-(1 / 2)}-\left.\frac{1}{2} \frac{\partial \tilde{\xi}^{m}}{\partial n}\right|_{n=N-1 / 2}, \\
& \xi_{N}^{m}=\left.\widetilde{\xi}^{m}\right|_{n=N-(1 / 2)}+\left.\frac{1}{2} \frac{\partial \widetilde{\xi}^{m}}{\partial n}\right|_{n=N-(1 / 2)} .
\end{aligned}
$$

Inserting $\Delta=\frac{1}{2}$ into Eq. (A6), the continuous PSD equation takes the form,

$$
\begin{aligned}
\frac{\partial \widetilde{\xi}^{m}}{\partial t}= & -\frac{\partial}{\partial n}\left[k _ { n - ( 1 / 2 ) } ^ { m } \left(\left(K_{n-(1 / 2)}^{m} c-1\right) \widetilde{\xi}^{m}\right.\right. \\
& \left.\left.-\frac{1}{2}\left(K_{n-(1 / 2)}^{m} c+1\right) \frac{\partial \tilde{\xi}^{m}}{\partial n}\right)\right] .
\end{aligned}
$$

In terms of the continuous cluster volume fraction function $y^{m}$, where $y^{m}(n, t)=n \widetilde{\xi}^{m}(n, t)$, Eq. (A12) takes the form

$$
\begin{aligned}
\frac{\partial y^{m}}{\partial t}= & -\frac{1}{n} \frac{\partial}{\partial n}\left[k _ { n - ( 1 / 2 ) } ^ { m } \left(\left\{\frac{1}{n}\left(K_{n-(1 / 2)}^{m} c-1\right)\right.\right.\right. \\
& \left.+\frac{1}{2 n^{2}}\left(K_{n-(1 / 2)}^{m} c+1\right)\right\} y^{m} \\
& \left.\left.-\frac{1}{2 n}\left(K_{n-(1 / 2)}^{m} c+1\right) \frac{\partial y^{m}}{\partial n}\right)\right] .
\end{aligned}
$$

Changing from $n$ to $x=\ln (n)$, the continuous equations for the PSD takes the form of Eq. (3.4).

\section{APPENDIX B: $\mathrm{SiO}_{2}$ DATA}

Rearranging Eqs. (4.1)-(4.4), the equilibrium constant for an $m, n$-cluster is given by

$$
\begin{aligned}
R T \ln \left(\frac{1}{K_{n}^{m}}\right)= & g_{n+1}^{m}-g_{n}^{m}-\mu_{\mathrm{SiO}_{2}(\mathrm{aq})}^{* *} \\
= & \psi(n) d_{m}+(1-\psi(n)) a_{m}-\mu_{\mathrm{SiO}_{2}(\mathrm{aq})}^{* *}+(1-\psi(n)) \\
& \times b_{m}\left[(n+1)^{2 / 3}-n^{2 / 3}\right] .
\end{aligned}
$$

For large crystals, $n \rightarrow \infty$, the solubility (i.e., $1 / K_{n}^{m}$ ) only depends on the bulk free energy via

$$
R T \ln \left(\frac{1}{K_{\infty}^{m}}\right)=R T \ln \left(c_{\infty}^{m}\right)=a_{m}-\mu_{\mathrm{SiO}_{2}(\mathrm{aq})}^{*},
$$

where $c_{\infty}^{m}$ is the concentration (mol/l) of $\mathrm{SiO}_{2}(\mathrm{aq})$ in equilibrium with bulk polymorph $m$. With this, the equilibrium constant of $m, n$-cluster becomes

$$
\begin{aligned}
\ln \left(K_{n}^{m}\right)= & -\ln \left(c_{\infty}^{m}\right)-\psi(n)\left(\frac{d_{m}-\mu_{\mathrm{Si}_{2}(\mathrm{aq})}^{* *}}{R T}-\ln \left(c_{\infty}^{m}\right)\right) \\
& -(1-\psi(n)) \frac{b_{m}}{R T}\left[(n+1)^{2 / 3}-n^{2 / 3}\right) .
\end{aligned}
$$

To calculate $K_{n}^{m}$ at a specific temperature, the bulk solubility $\left(c_{\infty}^{m}\right)$, surface free energy $\left(b_{m}\right)$, and adsorption energy $\left(d_{m}\right)$ of polymorph $m$ are needed. 

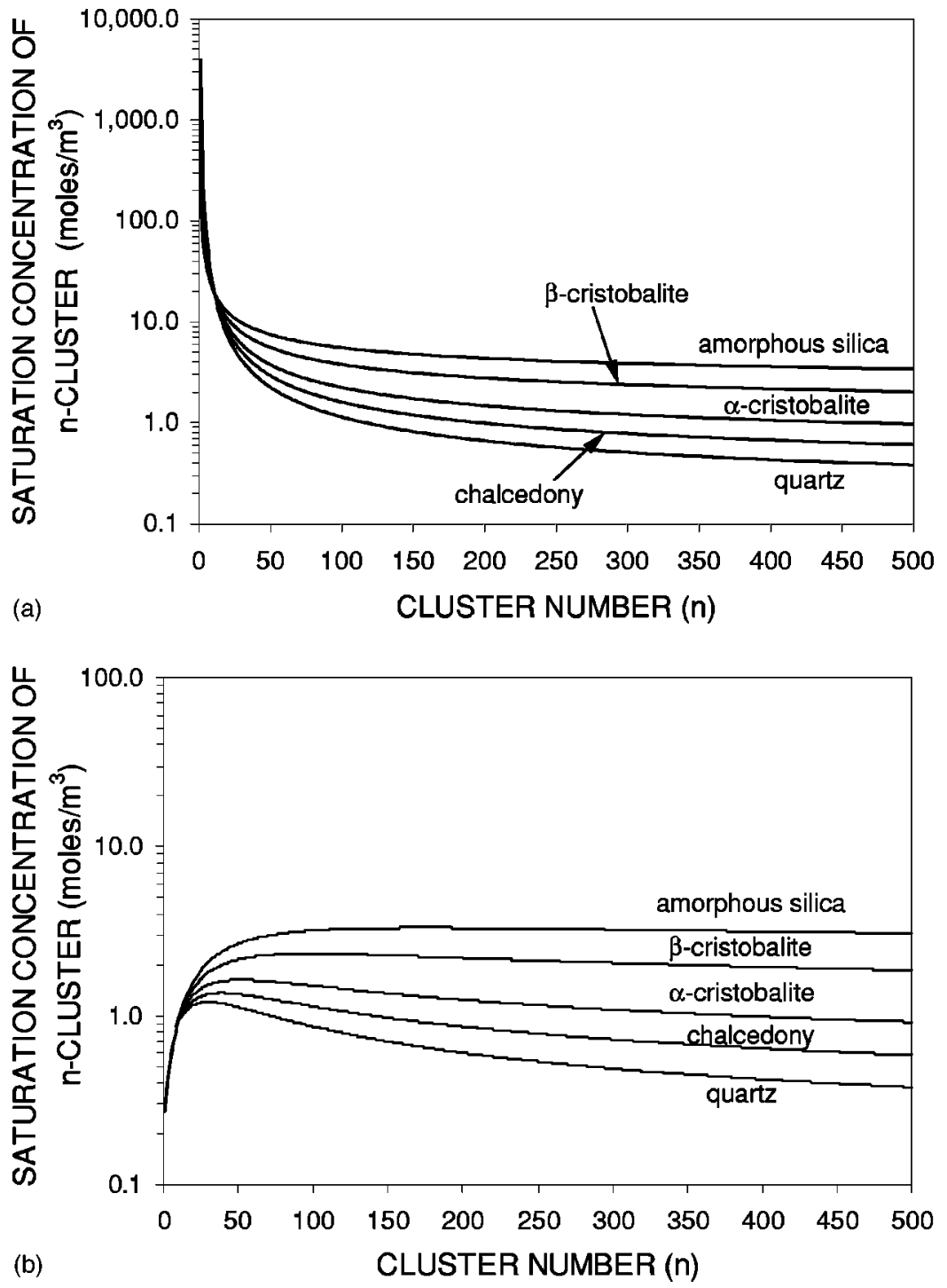

FIG. 12. Effect of adsorption energy on the equilibrium constants of silica polymorphs. The equilibrium constants vs cluster number at $20{ }^{\circ} \mathrm{C}$ for (a) no adsorption energy, and (b) $d_{m}=a^{\mathrm{qz}}$ for all polymorphs.

\section{Bulk solubility $\left(c_{\infty}^{m}\right)$}

Walther and Helgeson ${ }^{18}$ calculated bulk solubilities for silica polymorphs at temperatures ranging from 0 to $600{ }^{\circ} \mathrm{C}$ and these calculated solubilities are in agreement with experimentally derived values. In our numerical simulations, the bulk solubilities at a given temperature are calculated by polynomial approximation of the curves given by Walther and Helgeson. ${ }^{18}$

\section{Surface free energy $\left(b_{m}\right)$}

Where experimental data are available, surface free energies can be estimated using the observed dependence of solubility on specific surface area. These experiments have been reported for amorphous silica and quartz. ${ }^{17}$ If the $\mathrm{SiO}_{2}$ units on the surface of a grain partially project into the aqueous medium and partly reside in the bulk, then we might assume that the interfacial free energy is a weighted sum of the bulk and solution free energies; thus we conjecture

$$
\sigma_{m}=\left(A \mu_{\mathrm{SiO}_{2}(\text { aq })}^{* *}-B a_{m}\right) \frac{\Delta_{m}}{\bar{V}_{m}},
$$

where $\sigma_{m}=$ surface free energy of $m$ in contact with an aqueous solution; $\Delta_{m}=\left(\bar{V}_{m} / N^{0}\right)^{1 / 3}=$ lattice spacing normal to the surface (on the order of the thickness of the surface layer); $A=3.39643, B=0.53$.

The coefficients $A$ and $B$ were determined by substituting known $\sigma_{m}$ values for quartz and amorphous silica into Eq. (B4).

The surface free energy coefficient, $b_{m}$, is calculated from $\sigma_{m}$ via

$$
b_{m}=4 \pi \sigma_{m}\left(\frac{3 \bar{V}_{m}}{4 \pi N^{0}}\right)^{2 / 3} .
$$

\section{Adsorption energy $\left(d_{m}-\mu_{\mathrm{SiO}_{2}(\mathrm{aq})}^{* *}\right)$}

The kinetics of small clusters and nucleation initiation are strongly affected by the adsorption energy of $\mathrm{SiO}_{2}$ units to the site of nucleation. Clearly this quantity depends strongly on the atomic-scale geometry and chemical nature of the heterogeneous nucleation site. Values of $d_{m}$ are therefore most likely to be best estimated using molecular mechanics software and is beyond the scope of this study. How- 
ever, we have included this parameter in our study to investigate the sensitivity of the nucleation and Ostwald processes to this parameter. In Fig. 12, the effect of adsorption energy on saturation concentrations $\left(K_{n}^{m}\right)^{-1}$ are shown. In these figures, temperature is $20{ }^{\circ} \mathrm{C}$ and the diameter of the nucleation site is assumed to be $100 \AA$. If there is no adsorption energy, the initiation of quartz needs a huge supersaturation as seen in Fig. 12(a). Therefore, with no adsorption energy, at $20^{\circ} \mathrm{C}$ the initiation of quartz is practically impossible. In Fig. 12(b), the adsorption energies for all polymorphs are chosen to be the same, i.e., $d_{m}=a_{\mathrm{qz}}$. This selection enables the initiation of quartz, even at the saturation concentration of bulk quartz.

\section{Rate coefficients $\left(\overline{\boldsymbol{k}}_{m}\right)$}

We obtained rate coefficients from experimental data as follows. The rate of change of the number of $\mathrm{SiO}_{2}$ units in the solid phase per unit time is given by

$\frac{d}{d t}\left(\begin{array}{c}\text { number of units } \\ \text { in solid state per } \\ \text { unit system volume }\end{array}\right)$

$$
\begin{aligned}
& =\eta_{T} \sum_{m, n} n \frac{d \xi_{n}^{m}}{d t} \\
& =\eta_{T} \sum_{m, n} W_{n}^{m}=\eta_{T} \sum_{m, n} k_{n}^{m}\left[K_{n}^{m} c \xi_{n}^{m}-\xi_{n+1}^{m}\right],
\end{aligned}
$$

where the variables are the same as described in Sec. II. Consider a monomineralic, monodisperse system of particles of mineral $m$, where $\xi_{n}^{m}=0$ for $n \neq n^{\prime}, \xi_{n^{\prime}}^{m}=1$. When $n^{\prime}$ is sufficiently large, the rate coefficient varies slowly with respect to $n$, and thus, we can assume $k_{n^{\prime}-1}^{m} \approx k_{n^{\prime}}^{m}$. With these, the change in number of $\mathrm{SiO}_{2}$ in monodisperse systems becomes

$$
\frac{d}{d t}\left(\begin{array}{c}
\text { number of units } \\
\text { in solid state per } \\
\text { unit system volume }
\end{array}\right)=\eta_{T} k_{n^{\prime}}^{m}\left(K_{n^{\prime}}^{m} c-1\right) .
$$

From experimental data (see Refs. 19 and 23) the crystal growth rate is

$$
\frac{d r}{d t}=\bar{V}_{m} q_{m}\left(K_{n^{\prime}}^{m} c-1\right),
$$

where $q_{m}=$ experimental first order rate coefficient for dissolution $\left(\mathrm{mol} \mathrm{m}^{-2} \mathrm{~s}^{-1}\right) ; r=$ grain radius $(\mathrm{m})$.

The change of number of units in one grain of mineral $m$ per unit time is $\frac{d}{d t}\left(\begin{array}{c}\text { number of units } \\ \text { in one grain }\end{array}\right)$

$$
=\frac{N^{0}}{\bar{V}_{m}} 4 \pi r^{2} \frac{d r}{d t}=\left(\begin{array}{c}
\text { Surface area of } \\
\text { the grain }
\end{array}\right) N^{0} q_{m}\left(K_{n^{\prime}}^{m} c-1\right) .
$$

In the monomineralic, monodisperse system $\eta_{T}$ is the total number of grains per unit system volume, and, using $A_{n^{\prime}}^{m}$ for the surface area of an $n^{\prime}$ cluster, one finds

$$
\frac{d}{d t}\left(\begin{array}{c}
\text { number of units } \\
\text { in solid state per } \\
\text { unit system volume }
\end{array}\right)=\eta_{T} A_{n^{\prime}}^{m} N^{0} q_{m}\left(K_{n^{\prime}}^{m} c-1\right) .
$$

Comparing Eqs. (B7) and (B10) yields $k_{n^{\prime}}^{m}=A_{n^{\prime}}^{m} N^{0} q_{m}$. For sufficiently large, smooth clusters, the rate coefficient is independent from the adsorption effects the rate coefficient per unit area is related to the experimental dissolution rate via

$$
\bar{k}_{m}=q_{m} N^{0} .
$$

${ }^{1}$ P. Ortoleva, Geochemical Self-Organization (Oxford University Press, New York, 1994).

${ }^{2}$ J. W. Morse and W. M. Casey, Am. J. Sci. 288, 537 (1988).

${ }^{3}$ J. W. Morse and Q. Wang, Am. J. Sci. 296, 989 (1996).

${ }^{4}$ C. I. Steefel and P. Van Cappelen, Geochim. Cosmochim. Acta 54, 2657 (1990).

${ }^{5}$ K. Miyazaki, Geochim. Cosmochim. Acta 60, 277 (1996).

${ }^{6}$ W. D. Carlson, C. Denison, and R. A. Ketcham, Geological J. 30, 207 (1995).

${ }^{7}$ M. Tomellini, Appl. Surf. Sci. 99, 67 (1996).

${ }^{8}$ R. R. Bitti and P. E. di Nunzio, Scr. Mater. 39, 335 (1998).

${ }^{9}$ R. Poduri and L.-Q. Chen, Acta Mater. 46, 3915 (1998).

${ }^{10}$ I. M. Lifshitz and V. V. Slyozov, J. Phys. Chem. Solids 19, 35 (1961).

${ }^{11}$ C. Wagner, Ber. Bunsenges. Phys. Chem. 651, 581 (1961).

${ }^{12}$ A. J. Ardell, Acta Metall. 20, 61 (1972).

${ }^{13}$ M. Tsumuraya and Y. Miyate, J. Mater. Sci. 32, 2001 (1983).

${ }^{14}$ J. A. Marqusee and J. Ross, J. Chem. Phys. 80, 536 (1984).

${ }^{15}$ P. W. Voorhees, J. Stat. Phys. 38, 231 (1985).

${ }^{16}$ M. Tokuyama and K. Kawasaki, Physica A 123, 386 (1984).

${ }^{17}$ P. M. Dove, "Kinetic and thermodynamic controls on silica reactivity in weathering environments," in Chemical Weathering Rates of Silicate Minerals, Reviews in Mineralogy, edited by A. F. White and S. L. Brantley (Mineralogical Society of America, Washington, 1995), Vol. 31.

${ }^{18}$ J. V. Walther and H. C. Helgeson, Am. J. Sci. 277, 1315 (1977).

${ }^{19}$ J. D. Rimstidt and H. L. Barnes, Geochim. Cosmochim. Acta 44, 1683 (1980).

${ }^{20}$ C. A. J. Fletcher, Computational Techniques for Fluid Dynamics 2: Specific Techniques for Different Flow Categories (Springer-Verlag, Berlin, 1991).

${ }^{21}$ S. Kai, S. C. Muller, and J. Ross, J. Chem. Phys. 76, 1392 (1982).

${ }^{22}$ V. Nasreddine and R. Sultan, J. Phys. Chem. A 103, 2934 (1999).

${ }^{23}$ P. J. N. Renders, C. H. Gammons, and H. L. Barnes, Geochim. Cosmochim. Acta 59, 77 (1995). 